\title{
Disorder-induced stiffness degradation of highly disordered porous
} materials

\author{
Hadrien Laubie ${ }^{\mathrm{a}}$, Siavash Monfared ${ }^{\mathrm{a}}$, Farhang Radjaï ${ }^{\mathrm{b}, \mathrm{c}}$, Roland Pellenq ${ }^{\mathrm{a}, \mathrm{b}, \mathrm{d}}$, \\ Franz-Josef Ulm a, b, * \\ a Department of Civil and Environmental Engineering, Massachusetts Institute of Technology, Cambridge, MA 02139, USA \\ b $\langle\mathrm{MSE}\rangle{ }^{2}$, UMI 3466 CNRS - MIT Energy Initiative, Massachusetts Institute of Technology, 77 Massachusetts Avenue, Cambridge 02139, \\ USA \\ ${ }^{\mathrm{c}}$ LMGC, UMR 5508 CNRS - Université de Montpellier, 163 rue Auguste Broussonnet, 34090 Montpellier, France \\ ${ }^{\mathrm{d}}$ CINaM, CNRS - Aix Marseille Université, Campus de Luminy, 13288 Marseille Cedex 09, France
}

\section{A B S T R A C T}

The effective mechanical behavior of multiphase solid materials is generally modeled by means of homogenization techniques that account for phase volume fractions and elas- tic moduli without considering the spatial distribution of the different phases. By means of extensive numerical simulations of randomly generated porous materials using the lat- tice element method, the role of local textural properties on the effective elastic proper- ties of disordered porous materials is investigated and compared with different continuum micromechanics-based models. It is found that the pronounced disorder-induced stiffness degradation originates from stress concentrations around pore clusters in highly disordered porous materials. We identify a single disorder parameter, $\varphi s_{a}$, which combines a measure of the spatial disorder of pores (the clustering index, $s_{a}$ ) with the pore volume fraction (the porosity, $\varphi)$ to scale the disorder-induced stiffness degradation. Thus, we conclude that the classical continuum micromechanics models with one spherical pore phase, due to their underlying homogeneity assumption fall short of addressing the clustering effect, unless additional texture information is introduced, e.g. in form of the shift of the perco- lation threshold with disorder, or other functional relations between volume fractions and spatial disorder; as illustrated herein for a differential scheme model representative of a twophase (solid-pore) composite model material.

Keywords:

Inhomogeneous material

Porous material

Elastic material

Microstructures

Stress concentrations

\section{Introduction}

What is the effect of disorder on the effective elastic behavior of porous materials? - The question is of some relevance for porous materials whose effective mechanical behavior defies classical descriptors based on continuum micromechanics theory (for a review, see e.g. Suquet, 1997; Zaoui, 2002). Indeed, based primarily upon Eshelby's inclusion problem (Eshelby, 1957) and the assumption of scale separation, the consideration of composite materials as an assembly of (interacting) monodisperse spherical inclusions exhibiting characteristic morphologies from matrix-inclusion (Mori and Tanaka, 1973) to

\footnotetext{
* Corresponding author.

E-mail addresses: hlaubie@mit.edu (H. Laubie), monfared@mit.edu (S. Monfared), fradjai@mit.edu (F. Radjaï), pellenq@mit.edu (R. Pellenq), ulm@mit.edu (F.-J. Ulm).
} 


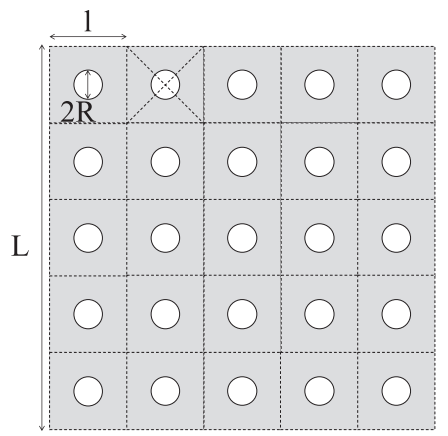

(a)

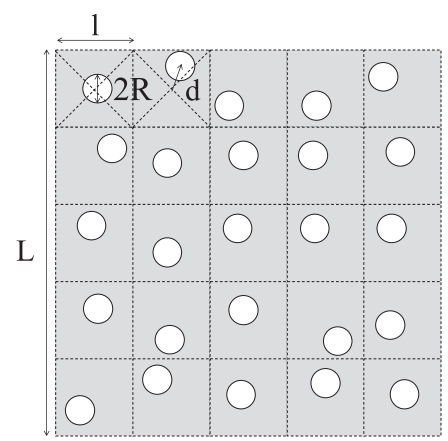

(b)

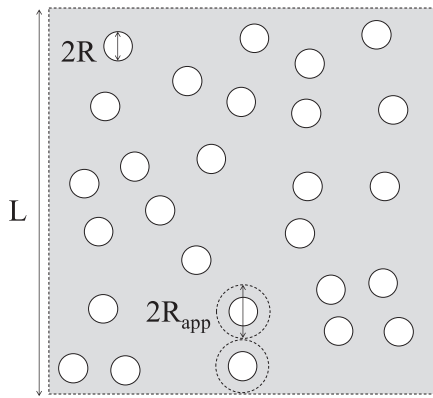

(c)

Fig. 1. Two-dimensional porous media: (a) ordered system, (b) type 1 disorder (pores confined in their unit cell), (c) type 2 disorder with $\lambda=R_{a p p} / R$ (here $\lambda>1)$.

poly-crystals and granular (Hill, 1965), fails to address explicitly mesoscale texture effects. Such texture effects originate often from the material manufacturing process (such as inhomogeneous precipitation in cement hydration Del Gado et al., 2014; Ioannidou et al., 2016; Masoero et al., 2012) or material maturation processes (such as biologically mediated inorganicorganic tissue growth (Hellmich and Ulm, 2002) or the diagenesis of organic-rich, naturally occurring porous geocomposites (Monfared and Ulm, 2016)).

Some studies have addressed the mesotextural effects associated with the non-spherical shape of inclusions seen in some geo- or bio-composites where the load-bearing phase can be modeled as an arrangement of randomly oriented single crystals that can have different aspect ratios. These studies include finite element analysis (see e.g. Meille and Garboczi, 2001 ) or theoretical analysis using self-consistent schemes adapted to the specific case of non-spherical inclusions (see e.g. Fritsch et al., 2006; 2009; 2010; 2017; Sanahuja et al., 2010).

We address here another mesotexture effect of porous materials: the local fluctuation of porosity around a mean value in materials having a matrix (solid)/inclusions (pores) morphology. While porous material systems with both small and large fluctuations have been studied both theoretically and experimentally, a search of the relevant literature was not conclusive in finding a comprehensive investigation that bridges the two asymptotes for a wide range of porosities, and thus remains to be developed. This is in short the focus of this paper. In fact, for small local fluctuations, analytical expressions based on asymptotic expansion for isotropic porous materials show a disorder-induced compliance increase (i.e. stiffness degradation) (Gărăjeu and Suquet, 2007). On the other end of the spectrum, experimental results by Lobb and Forrester (1987) of highly disordered 2-D porous systems (obtained by perforating square metal sheets with holes at random positions that could overlap or miss each other by any amount, thus exhibiting large porosity fluctuations), confirm the persistent effect of disorder on the effective elasticity; and highlight the need to bridge the gap between small and large porosity fluctuation systems.

This will be achieved, in this paper, by first generating a large range of disordered porous materials by means of canonical Monte Carlo moves on hard-disks/spheres exhibiting different porosities. The elasticity of these systems is then investigated by means of the Lattice Element Method, with the focus on identifying an appropriate 'order' or 'disorder' parameter that is able to consistently scale disorder-induced stiffness degradation. Finally, we suggest a simple method to integrate this scaling into a conventional continuum-based micromechanics model.

\section{Materials and methods}

The focus of the current investigation is the elasticity of porous material systems exhibiting different levels of disorder. This requires the generation of a large range of porous material samples with controlled disorder. This is achieved here by considering deviations from a periodic arrangement of pores in 2-D and 3-D, taken as reference, and by quantifying the disorder-induced stiffness degradation with respect to the reference elasticity of the ordered system.

\subsection{Porous sample generation. Disorder characterization}

A periodic porosity arrangement (periodicity $l$ ) for both 2-D and 3-D porous material systems is considered as reference. In 2-D, this is achieved in form of $N$ disk-shaped pores of radius $R$ (Fig. 1(a)) placed in a square plate (matrix phase) of size $L_{x}=L_{z}=L=\sqrt{N} l$, and thickness $L_{y}=d \ll L$; while, in 3-D, spherical pores of radius $R$ are placed in a cubic matrix of size $L_{x}=L_{y}=L_{z}=L=\sqrt[3]{N} l$. The porosity $\varphi$ of these systems is tuned by varying the size $R$ of the pores and/or their number $N$ :

$$
\left\{\begin{array}{rl}
\varphi^{\text {disk }} & =N \pi\left(\frac{R}{L}\right)^{2}=\pi\left(\frac{R}{l}\right)^{2} \\
\varphi^{\text {sphere }} & =N\left[\frac{4}{3} \pi\left(\frac{R}{L}\right)^{3}-\frac{6}{N} v(R)\right]=\frac{4}{3} \pi\left(\frac{R}{l}\right)^{3}-6 v(R)
\end{array},\right.
$$


with $v(R)$ a dimensionless function defined by (see Appendix A):

$$
v(R)= \begin{cases}0, & \text { if } R \leq l / 2 \\ \frac{\pi}{24}\left(\frac{2 R}{l}-1\right)^{2}\left(\frac{4 R}{l}+1\right), & \text { otherwise. }\end{cases}
$$

Disorder is introduced into this periodic arrangement by different methods:

- Type 1: Random moves within a unit cell (Fig. 1(b)). Pores (disks or spheres of radius $R$ ) of the ordered systems are moved to random positions at a distance $0<d<l / 2-R$ contained within their original unit cell (defined by the periodicity $l$ ). The number $N$ of pores is kept fixed and the porosity increases as the pore radius $R$ is increased.

- Type 2: Monte-Carlo moves at constant number $N$ of pores of respectively hard-disks in 2-D and spheres in 3-D with an apparent pore radius $R_{a p p}=\lambda R$. With $\lambda \in[1, l /(2 R)]$, the generated systems contain only non-overlapping pores of an overall porosity that increases with the pore radius, $R$. This type of Monte-Carlo generation -at constant $N$ - includes as a subset the type 1 generation method. But it is more general as the pore movement is not confined to the unit cell (see Fig. 1(c)), thus permitting larger texture deviations from the quasi-ordered system, $\lambda=l /(2 R)$, to highly disordered systems, $\lambda=1$.

- Type 3: Grand Canonical Monte Carlo (GCMC) insertion with variable number $N$ of pores of fixed apparent pore radius $R_{a p p}=\lambda R$, where $\lambda \in[0,1]$ defines an arbitrary degree of impenetrability (Smith and Torquato, 1988): GCMC with $\lambda=1$ corresponds to the hard-disk/hard-sphere porous model; while GCMC with $\lambda=0$ corresponds to fully penetrable disks and spheres (overlapping pores). In these GCMC-based generations, the porosity is defined by the number $N$ of pores, eventually corrected for the level of overlapping.

The methods thus described generate a large range of different disordered microstructures, even at same mean porosity. To illustrate this range of disorder, different descriptors classically employed in the characterization of microstructure (Torquato, 2002) are used. One classical descriptor of a two-phase (solid-porosity) microstructure is the two-point probability function of the pore phase, $S_{2}(r)$, which defines the probability that two points separated by a distance $r$ are both in a pore:

$$
S_{2}\left(r=\left\|\vec{r}_{2}-\vec{r}_{1}\right\|\right)=\left\langle I\left(\vec{r}_{1}\right) I\left(\vec{r}_{2}\right)\right\rangle,
$$

where the characteristic function $I\left(\vec{r}_{i}\right)=1$ if $\vec{r}_{i}$ is in the pore, and $I\left(\vec{r}_{i}\right)=0$ otherwise; whereas angular brackets denote ensemble average. While the one-point probability function defines the mean-porosity, $\varphi$; i.e.

$$
S_{1}\left(\vec{r}_{i}\right)=\left\langle I\left(\vec{r}_{i}\right)\right\rangle=\varphi,
$$

the two-point probability function, $S_{2}(r)$, exhibits the asymptotic properties (Smith and Torquato, 1988):

$$
\left\{\begin{array}{rl}
S_{2}(0) & =\varphi \\
\lim _{r \rightarrow \infty} S_{2}(r) & =\varphi^{2} \\
\left.\frac{d S_{2}}{d r}\right|_{r=0} & =-\frac{\varphi}{l_{c}}
\end{array},\right.
$$

with $l_{c}$ the mean chord length $\left(l_{c}=2 R\right.$ in the case of disk-shaped or spherical pores). The two-point probability function of four different systems, exhibiting the same mean porosity, are shown in Fig. 2 with their respective microstructure. For quasi-ordered systems (Fig. 2 (a), generated with $\lambda=l /(2 R)$ ), clear peaks appear with a fixed periodicity. As disorder increases (Fig. 2(b) and (c), $\lambda \rightarrow 1$ ), long-range peaks progressively disappear. When overlapping of disks/spheres is allowed (Fig. $2(\mathrm{~d}), \lambda=0$ ), the two-point probability function is almost flat after a quasi-linear decrease from $\varphi$ to $\varphi^{2}$.

The second descriptor employed is the Probability Distribution Function (PDF) of the local porosity throughout the sample, $f_{\varphi}$. It is obtained by measuring the local porosity $\varphi_{a}$ in a square-shaped control volume of side-length $a$ (here, $a=\sqrt{\pi R^{2} / \varphi}$ in 2-D and $a=\sqrt[3]{4 / 3 \pi R^{3} / \varphi}$ in 3-D for $\varphi \leq \pi / 6$ ). Fig. 3 displays the thus obtained PDFs for the four microtextures of the same average porosity, $\varphi=\left\langle\varphi_{a}\right\rangle$, considered before. While quasi-ordered systems (Fig. $\left.3(\mathrm{a}), \lambda=l /(2 R)\right)$ exhibit a narrow distribution with a clear peak centered around the average porosity, the probability density function broadens as disorder increases (Fig. 3(b) and (c), $\lambda \rightarrow 1$ ). With further disorder introduced by overlapping disks/spheres (Fig. 3(d), $\lambda=0$ ), the PDF exhibits a two-peak structure reminiscent of a solid-pore space phase separation akin to demixing, with a narrow peak around $\varphi_{a} \rightarrow 0$ (solid), and a diffuse peak around the average porosity. Analogous to the mixing index used to determine the degree of mixture of particulate materials (see e.g. Lacey, 1954), this spread of the local porosity $\varphi_{a}$ is captured -in first order- by a clustering index $s_{a}$, defined as the standard deviation of the porosity; that is, in a continuum form:

$$
s_{a}=\sqrt{\left\langle\left(\varphi_{a}-\varphi\right)^{2}\right\rangle}=\sqrt{\left\langle\varphi_{a}^{2}\right\rangle-\left\langle\varphi_{a}\right\rangle^{2}},
$$



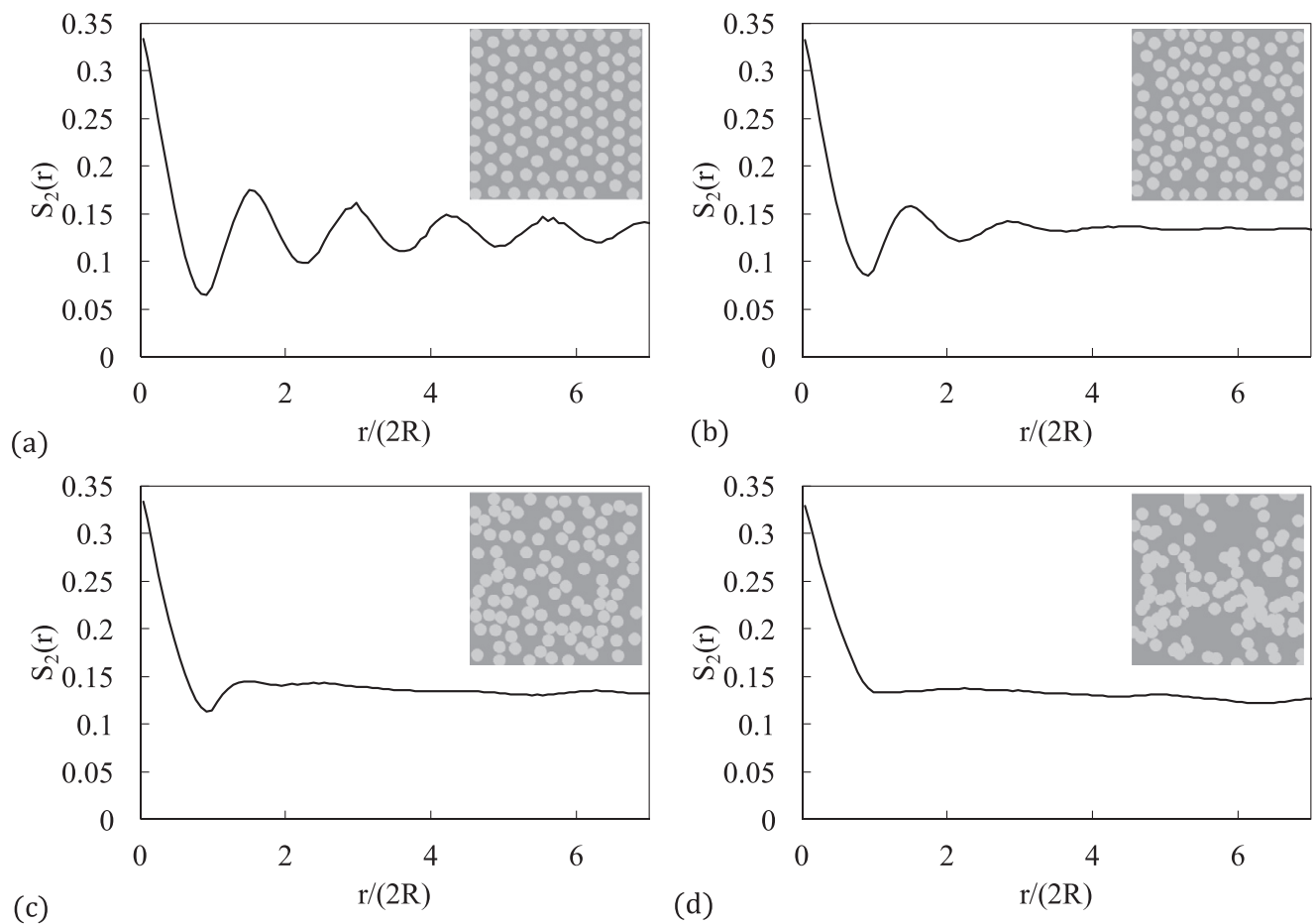

Fig. 2. Two-point probability functions and associated microstructures of four systems of increasing disorder $(\varphi=0.36)$ : (a) type 2 disorder with $\lambda=l /(2 R)$ (quasi-ordered system), (b) type 2 disorder with $\lambda=(l /(2 R)+1) / 2$, (c) type 2 disorder with $\lambda=1$ and (d) type 3 disorder with $\lambda=0$ (overlapping disks). Note the asymptotic values: $S_{2}(r \rightarrow 0)=\varphi=0.36$ and $S_{2}(r \rightarrow \infty)=\varphi^{2}=0.13$.
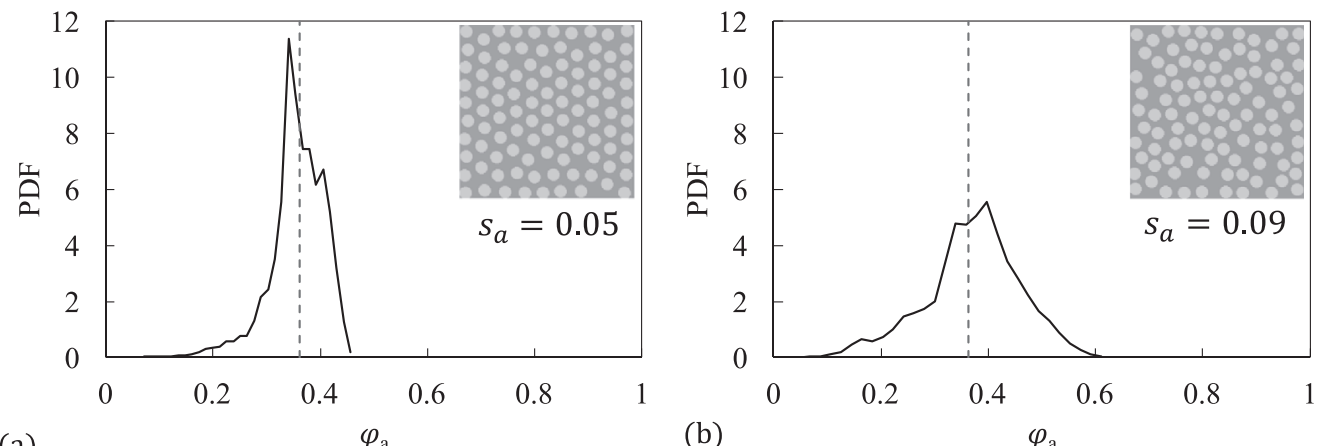

(a)

(b)
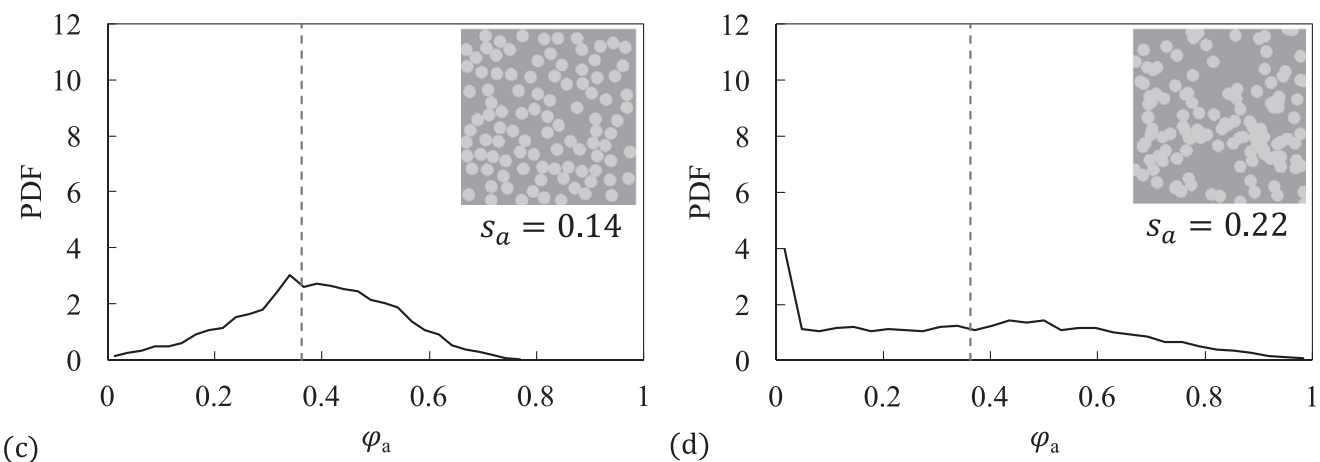

Fig. 3. Probability Density Function (PDF) of the local porosity $\left(\varphi_{a}\right)$ and associated microstructure for the same four systems (Fig. 2). The vertical line corresponds to the average porosity $(\varphi)$. 


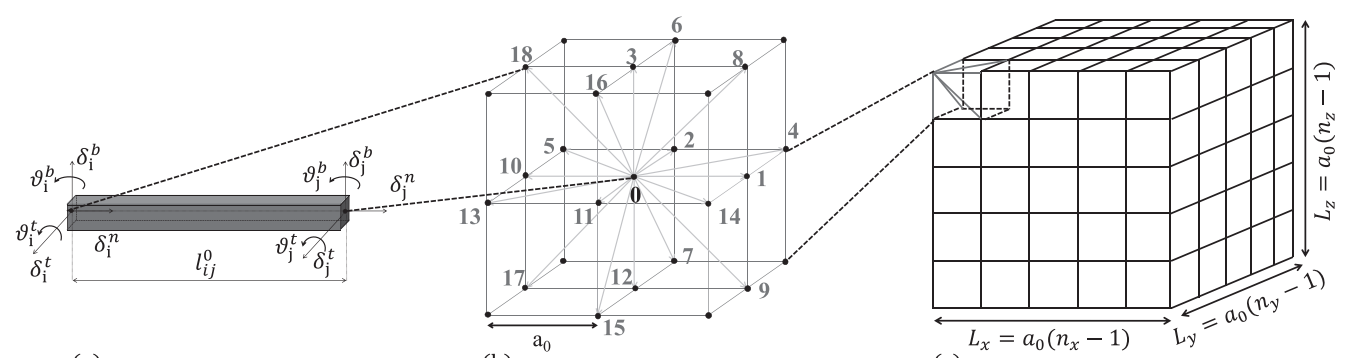

(a)

(b)

(c)

Fig. 4. (a) Degrees of freedom of a link element between points $i$ and $j$, (b) D3Q18 unit cell, (c) simulation box.

or, in a discrete form as the corrected standard deviation of the porosity:

$$
s_{a} \simeq \sqrt{\frac{\sum_{i=1}^{N_{a}}\left(\varphi_{a}^{i}-\varphi\right)^{2}}{N_{a}-1}},
$$

where $\varphi$ is the average sample porosity; $\varphi_{a}^{i}$ is the local porosity around a randomly chosen point $i, i \leq N_{a}$ with $N_{a}$ large enough to fully sample the specimen studied. Thus, $s_{a}=0$ corresponds to a perfectly ordered system, exhibiting no variability in the local porosity; whereas large values of $s_{a}$ correspond to segregated systems in which some degree of clustering exists in the pore configuration. The clustering index increases with the width of the probability density functions. By way of example, the clustering indexes of the PDFs shown in Fig. 3, increase from $s_{a}=0.05$ for the quasi-ordered system (Fig. 3(a)) to $s_{a}=0.09$ and $s_{a}=0.14$ for non-overlapping disks (Fig. 3(b) and c), and reaches the highest value, $s_{a}=0.22$, for overlapping disks (Fig. 3(d)). For purpose of completeness, we note that the clustering index is closely related to the coarseness $C=s_{a} /(1-\varphi)$ introduced by Torquato and coworkers (Lu and Torquato, 1990; Torquato, 2002). They proved that the coarseness $C$ was directly related to the two-point probability function $S_{2}(r)$ (see Appendix B in the case of fully penetrable disks). The clustering index $s_{a}$ defined by Eqs. (6) and (7) will be of some help in delineating the effect of disorder on the elasticity properties of the generated porous samples.

\subsection{Effective stiffness measure using the lattice element method}

The second tool employed in this investigation of the effect of disorder on mechanical properties of porous materials is the Lattice Element Method (LEM) (see e.g. Herrmann and Roux, 1990; Topin et al., 2007 and references herein). The generated porous mesostructures are discretized in a number of mass points representing the solid or the pore domain. Much akin to Potential of Mean Force (PMF) approaches used in Soft Matter Physics (see e.g. Masoero et al., 2012), these mass points interact with their nearest neighbors through effective interaction potentials (Laubie et al., 2017a). In this approach (Fig. 4), the reference configuration consists of $N=n_{x} n_{y} n_{z}$ mass points on a cubic lattice (of unit cell size $a_{0}$ ), having six degrees of freedom: three translations $\vec{\delta}$ and three rotations $\vec{\vartheta}$. Each mass point $i$ (reference position $\vec{x}_{i}$ ) interacts with a fixed number of neighboring points $j$ (a maximum of 18 in this study, corresponding to a cut-off radius $r_{\text {cutof } f}=\sqrt{2} a_{0}$ in PMF-approaches) via the potential:

$$
U_{i j}=U_{i j}^{s}+U_{i j}^{b},
$$

where $U_{i j}^{s}$ is a stretch term and $U_{i j}^{b}$ a bending term. With a focus on the elasticity of the samples close to their equilibrium configuration, the interaction potentials in the solid domain are approximated by harmonic expressions:

$$
\left\{\begin{array}{rl}
U_{i j}^{s}= & \frac{1}{2} \epsilon_{i j}^{n}\left(\frac{\delta_{j}^{n}-\delta_{i}^{n}}{l_{i j}^{0}}\right)^{2} \\
U_{i j}^{b}= & \frac{1}{2} \epsilon_{i j}^{t}\left\{\left(\frac{\delta_{j}^{b}-\delta_{i}^{b}}{l_{i j}^{0}}-\vartheta_{i}^{t}\right)^{2}+\left(\frac{\delta_{j}^{t}-\delta_{i}^{t}}{l_{i j}^{0}}+\vartheta_{i}^{b}\right)^{2}\right. \\
& +\left(\frac{\delta_{j}^{b}-\delta_{i}^{b}}{l_{i j}^{0}}-\vartheta_{i}^{t}\right)\left(\vartheta_{i}^{t}-\vartheta_{j}^{t}\right)+\left(\frac{\delta_{j}^{t}-\delta_{i}^{t}}{l_{i j}^{0}}+\vartheta_{i}^{b}\right)\left(\vartheta_{j}^{b}-\vartheta_{i}^{b}\right) \\
& \left.+\frac{1}{3}\left(\left(\vartheta_{j}^{b}-\vartheta_{i}^{b}\right)^{2}+\left(\vartheta_{i}^{t}-\vartheta_{j}^{t}\right)^{2}\right)\right\}
\end{array} .\right.
$$

Herein $l_{i j}^{0}=\left\|\vec{r}_{i j}\right\|$ (with $\vec{r}_{i j}=\vec{x}_{j}-\vec{x}_{i}=l_{i j}^{0} \vec{e}_{n}$ ) is the distance between solid mass points $i$ and $j$ in the reference configuration, while the solid's energy parameters $\epsilon_{i j}^{n, t} \sim a_{0}^{3} E_{s}$ are calibrated to recover the desired effective (or macroscopic) elastic 
behavior of the (homogeneous) solid phase ( $\varphi=0$; with Young's modulus $E_{S}$ and Poisson's ratio $v_{s}$ ), following the procedure outlined in Laubie et al. (2017a). If only the stretch term is considered (setting $\epsilon_{i j}^{t}=0$ ), the value of the Poisson's ratio of the composite is defined by the geometric limit value of the cubic lattice; that is $v_{\lim }=1 /(D+1)$ (with $D$ the space dimension). The bending term is required to model materials with lower Poisson's ratios, $v \leq v_{\text {lim. }}$. The conjugated forces to translational degrees of freedoms derive from the potential:

$$
\vec{F}_{i}^{j}=-\frac{\partial U_{i j}}{\partial \vec{\delta}_{i}} .
$$

The stress measure at each mass point is obtained using the virial expression:

$$
\sigma_{i}=\frac{1}{2 V_{i}} \sum_{j=1}^{N_{i}^{b}} \vec{r}_{i j} \otimes \vec{F}_{i}^{j},
$$

with $V_{i}=a_{0}^{3}$ the volume of the unit cell, and $N_{i}^{b}$ the number of point $i$ 's neighboring mass points. The total (or average) stress in volume $V=\left(n_{x}-1\right)\left(n_{y}-1\right)\left(n_{z}-1\right) a_{0}^{3}$ is:

$$
\langle\boldsymbol{\sigma}\rangle=\frac{1}{V} \sum_{i=1}^{N} V_{i} \sigma_{i} .
$$

Finally, all links belonging to the pore region have zero energy parameters, $\epsilon_{i j}^{n, t}=0$; and thus zero-forces $\left(\vec{F}_{i}^{j}=0\right)$ and stresses $\left(\sigma_{i}=0\right)$. For an extension of the LEM approach to linear poromechanics, see Monfared et al. (2017).

In order to measure the effective stiffness (in tension) of the different systems studied, a displacement is prescribed on the boundaries; that is $\overrightarrow{\delta_{-}}=-\delta / 2 \vec{e}_{x}$ on mass points on surface $x=0$ of the structure, and $\overrightarrow{\delta_{+}}=\delta / 2 \vec{e}_{x}$ on mass points on surface $x=L=\left(n_{x}-1\right) a_{0}$. The lateral boundaries of the structure are force free. After relaxation, i.e. after minimization of the potential energy, $\mathcal{E}_{\text {pot }}=\min _{\vec{\delta}_{i}, \vec{\vartheta}_{i}} \sum_{\text {links } k l} U_{k l}$, with respect to both the translational and rotational degrees of freedom, the effective stiffness is obtained from:

$$
E_{e f f}=\left\langle\sigma_{x x}\right\rangle \frac{L}{\delta},
$$

where $\left\langle\sigma_{x x}\right\rangle=\vec{e}_{x} \cdot\langle\sigma\rangle \cdot \vec{e}_{x}$ is the axial stress and $\delta \ll L$.

\subsection{Continuum micromechanics solutions}

The discrete solutions will be bench-marked against continuum micromechanics solutions that explicitly address the effect of disorder on elasticity of heterogeneous materials; by considering local porosity fluctuations of the form (Gărăjeu and Suquet, 2007):

$$
\varphi_{t}(\vec{x})=\varphi+t \delta_{\varphi}(\vec{x})
$$

with $\left\langle\varphi_{t}(\vec{x})\right\rangle=\varphi$ and $\left\langle\delta_{\varphi}\right\rangle=0$. The standard deviation of the local porosity is obtained from an application of Eq. (6) (except that there is no observation window size $a$ defined here):

$$
\sigma_{\varphi}^{2}=\left\langle\left(\varphi_{t}-\varphi\right)^{2}\right\rangle=t^{2}\left\langle\delta_{\varphi}^{2}\right\rangle .
$$

The two benchmark models herein considered are (1) the asymptotic expansion model of Gărăjeu and Suquet (2007), considering small fluctuations $t \ll 1$; and (2) a differential scheme model inspired by Norris (1985) where a composite porous solid is built incrementally by a series of addition of different phases having different elastic moduli.

To simplify the presentation, a $\mathcal{N}$-phase composite with phase $i$ (concentration $\Phi_{i}, \sum_{i=1}^{\mathcal{N}} \Phi_{i}=1$ ) occupying the volume $\Omega_{i}$ with $i \in\{1, \ldots, \mathcal{N}\}$ is considered. The local porosity is homogeneous in the subvolumes, i.e. $\varphi_{t}(\vec{x})=\varphi+t \delta_{\varphi}^{(i)}$ for $\vec{x} \in \Omega_{i}$.

\subsubsection{Mori-Tanaka-based asymptotic expansion model}

The asymptotic expansion model of Gărăjeu and Suquet (2007) departs from the classical reference solution of an ordered porous material morphology $\left(\sigma_{\varphi}=0\right)$; that is, the matrix-pore inclusion morphology exemplified by the Mori-Tanaka scheme $^{1}$ (superscript $M T$ ):

$$
\mathbf{C}^{M T}=(1-\varphi) \mathbf{C}^{s}:\left((1-\varphi) \mathbf{I}+\varphi(\mathbf{I}-\mathbf{S})^{-1}\right)^{-1},
$$

\footnotetext{
${ }^{1}$ Although the Mori-Tanaka scheme was originally derived for random microstructures, it captures fairly well the behavior of periodic (ordered) systems (see Section 3.1).
} 
where $\mathbf{I}$ is the fourth-order unit tensor and $\mathbf{C}^{s}$ is the solid's stiffness tensor, and $\mathbf{S}$ is the Eshelby tensor, which for 2-D cylindrical pores in an isotropic matrix (Dormieux et al., 2006) and plane-stress conditions permits the following specification of the Young's modulus:

$$
\frac{E_{M T}^{2 D}(\varphi)}{E_{s}}=\frac{1-\varphi}{1+2\left(1-v_{s}^{2}\right) \varphi},
$$

and for 3-D spherical pores in an isotropic matrix (Dormieux et al., 2006):

$$
\frac{E_{M T}^{3 D}(\varphi)}{E_{s}}=\frac{1-\varphi}{1+\frac{\left(1+v_{s}\right)\left(13-15 v_{s}\right)}{2\left(7-5 v_{s}\right)} \varphi},
$$

with $E_{s}$ and $v_{s}$ the solid's Young's modulus and Poisson's ratio, respectively. The following classical isotropic elasticity equations were herein used:

$$
\left\{\begin{array}{l}
E_{M T}^{2 D}(\varphi)=C_{11}^{2 D, M T}(\varphi)\left(1-\left(\frac{C_{12}^{2 D, M T}(\varphi)}{C_{11}^{2 D, M T}(\varphi)}\right)^{2}\right) \\
E_{M T}^{3 D}(\varphi)=C_{11}^{3 D, M T}(\varphi)\left(1-\frac{2\left(C_{12}^{3 D, M T}(\varphi)\right)^{2}}{\left(C_{11}^{3 D, M T}(\varphi)\right)^{2}+C_{11}^{3 D, M T}(\varphi) C_{12}^{3 D, M T}(\varphi)}\right)
\end{array}\right.
$$
$\epsilon$ :

Thus, for reference, the elastic energy $\tilde{w}(\varphi, \epsilon)$ of the ordered system $\left(\sigma_{\varphi}=0\right)$ is a quadratic function of the strain tensor

$$
\tilde{w}(\varphi, \boldsymbol{\epsilon})=\frac{1}{2} \boldsymbol{\epsilon}: \mathbf{C}^{M T}(\varphi): \boldsymbol{\epsilon}
$$

In return, when fluctuations in porosity are considered $\left(\sigma_{\varphi} \neq 0\right)$, this elastic energy becomes ${ }^{2}$ :

$$
\tilde{\tilde{w}}(t, \overline{\boldsymbol{\epsilon}})=\left\langle\tilde{w}\left(\varphi_{t}, \boldsymbol{\epsilon}_{t}\right)\right\rangle
$$

The asymptotic expansion of this energy in $t$ reads (Gǎrǎjeu and Suquet, 2007):

$$
\tilde{\tilde{w}}(t, \overline{\boldsymbol{\epsilon}})=\tilde{w}(\varphi, \overline{\boldsymbol{\epsilon}})+\frac{t^{2}}{2}\left(\left\langle\delta_{\varphi}^{2}\right\rangle \frac{\partial^{2} \tilde{w}}{\partial \varphi^{2}}(\overline{\boldsymbol{\epsilon}})-\frac{\partial^{2} \tilde{w}}{\partial \varphi \partial \boldsymbol{\epsilon}}(\overline{\boldsymbol{\epsilon}}): \mathbf{H}: \frac{\partial^{2} \tilde{w}}{\partial \varphi \partial \boldsymbol{\epsilon}}(\overline{\boldsymbol{\epsilon}})\right)+O\left(t^{3}\right),
$$

where $\mathbf{H}$ is a fourth-order tensor. For a two-phase system $(\mathcal{N}=2)$ with $\delta_{\varphi}^{(1)}=-\Delta \varphi / \Phi_{1}$ and $\delta_{\varphi}^{(2)}=\Delta \varphi / \Phi_{2}$ so that $\left\langle\delta_{\varphi}\right\rangle=$ $\Phi_{1} \delta_{\varphi}^{(1)}+\Phi_{2} \delta_{\varphi}^{(2)}=0$ and $\left\langle\delta_{\varphi}^{2}\right\rangle=(\Delta \varphi)^{2} /\left(\Phi_{1} \Phi_{2}\right)$, Gărăjeu and Suquet (2007) showed that the H-tensor reduces to $\mathbf{H}=\left\langle\delta_{\varphi}^{2}\right\rangle \mathbf{S}$ : $\left(\mathbf{C}^{M T}\right)^{-1}$ with $\mathbf{S}$ the Eshelby tensor of the ordered (matrix-inclusion) reference system, as employed in Eq. (16). Using this result, while replacing in Eq. (22) $t^{2}\left\langle\delta_{\varphi}^{2}\right\rangle$ by the porosity standard deviation $\sigma_{\varphi}^{2}$ according to Eq. (15), the elastic energy of the disordered system is recast in the form:

$$
\tilde{\tilde{w}}\left(\sigma_{\varphi}, \overline{\boldsymbol{\epsilon}}\right)=\frac{1}{2} \overline{\boldsymbol{\epsilon}}: \mathbf{C}^{e f f}\left(\sigma_{\varphi}\right): \overline{\boldsymbol{\epsilon}}+O\left(\sigma_{\varphi}^{3}\right)
$$

with $\mathbf{C}^{\text {eff }}$ the effective stiffness tensor:

$$
\mathbf{C}^{e f f}\left(\sigma_{\varphi}\right)=\mathbf{C}^{M T}+\frac{\sigma_{\varphi}^{2}}{2}\left(\frac{\partial^{2} \mathbf{C}^{M T}}{\partial \varphi^{2}}-2 \frac{\partial \mathbf{C}^{M T}}{\partial \varphi}: \mathbf{S}:\left(\mathbf{C}^{M T}\right)^{-1}: \frac{\partial \mathbf{C}^{M T}}{\partial \varphi}\right) .
$$

While expression (24) is somewhat involving, in that it does not permit simple closed-form solutions, it is readily implemented for the 2-D and 3-D matrix-inclusion morphologies, given by the Mori-Tanaka reference solution (16) and the corresponding Eshelby tensor expressions. Gărăjeu and Suquet (2007) presented this implementation through the consideration of spherical pores in an incompressible isotropic matrix. The extension of this solution to spherical pores in a compressible isotropic matrix is given in a closed form in Appendix C. Similar derivation were performed for the case of cylindrical pores, but do not permit simple closed-form solution. Provided such solution for $\mathbf{C}^{e f f}\left(\sigma_{\varphi}\right)$, the effective Young's modulus is obtained from (19), analogous to Eqs. (17) and (18).

Fig. 5 displays sample output, $E_{\text {eff }} / E_{s}$, for the asymptotic expansion model with $\Phi_{1}=\Phi_{2}=1 / 2$ and Poisson's ratio $v_{s}=$ $1 /(1+D)$ in D-dimension as a function of the porosity for different $\sigma_{\varphi}$ values (in 2-D, Fig. 5(a) and in 3-D, Fig. 5(b)). While some of the $\sigma_{\varphi}$ values may well be beyond the range of validity of the model, $t \ll 1$, the figures clearly highlight that a local variability of the porosity lowers the effective stiffness as well as the predicted percolation threshold. This effect is particularly pronounced at high porosity.

\footnotetext{
2 From now on, the dependence in $\varphi$ is dropped to simplify the notations. All the functions are implicitly evaluated at $\varphi_{t}=\varphi$.
} 

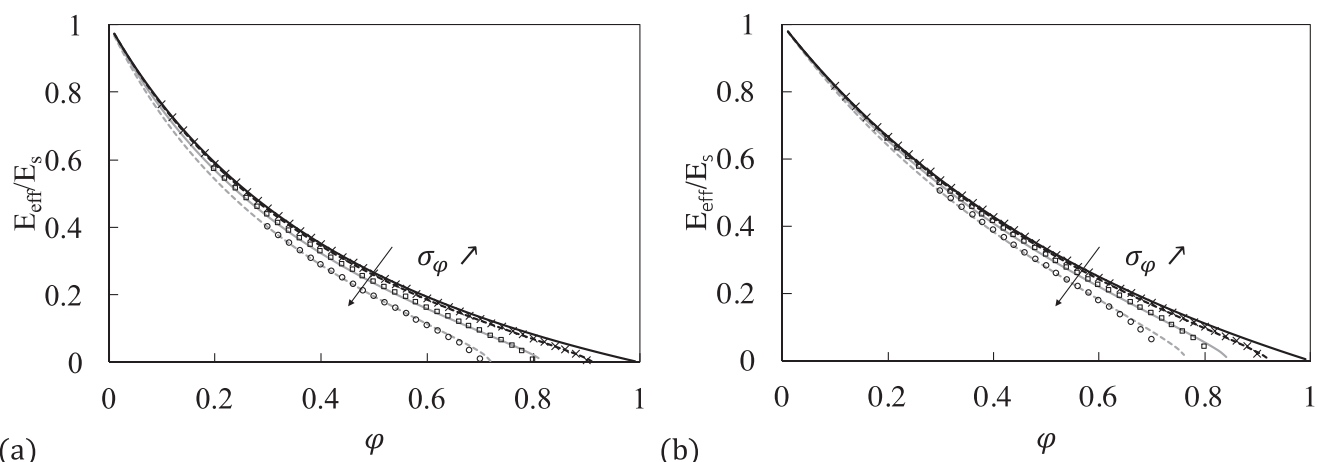

Fig. 5. Dimensionless effective Young's modulus: $E_{e f f}(\varphi) / E_{s}$ as function of the porosity in 2-D (a) and 3-D (b). Values obtained using the asymptotic development (24) (lines) and the differential scheme Eq. (26) (symbols) for $\sigma_{\varphi}=0$ (i.e. Mori-Tanaka scheme), $\sigma_{\varphi}=0.1, \sigma_{\varphi}=0.2$ and $\sigma_{\varphi}=0.3$.

\subsubsection{Differential scheme model to capture porosity fluctuations}

The second model herein considered is based on the differential scheme (Norris, 1985), in which a $\mathcal{N}$-phase model is built incrementally. Starting from a homogeneous elastic medium (bulk and shear moduli, $K_{0}$ and $G_{0}$, volume fraction $c_{0}(0)=1$ ), $i=1, \mathcal{N}$ phases (of bulk and shear moduli, $K_{i}$ and $G_{i}$, volume fraction $c_{i}(t)$ ) are successively added, while keeping the total volume constant, such that $c_{0}(t)+c(t)=1$ with $c(t)=\sum_{i=1}^{\mathcal{N}} c_{i}(t)$. The effective moduli $\left(K_{\text {eff }}, G_{\text {eff }}\right)$ are obtained from solving the coupled differential equations:

$$
\left\{\begin{array}{l}
\dot{K}^{e f f}=\sum_{i=1}^{\mathcal{N}}\left(K_{i}-K_{e f f}\right)\left(\dot{c}_{i}+\dot{c} \frac{c_{i}}{1-c}\right) P_{i} \\
\dot{G}^{e f f}=\sum_{i=1}^{\mathcal{N}}\left(G_{i}-G_{e f f}\right)\left(\dot{c}_{i}+\dot{c} \frac{c_{i}}{1-c}\right) Q_{i}
\end{array}\right.
$$

where the dot denotes time derivation, while coefficients $\left(P_{i}, Q_{i}\right)$ depend on the phase morphology as specified later on. It is readily understood, that the solution of the coupled differential equation (25) depends on the path chosen for $c_{i}(t)$. The path used here is such that the $c_{i} \mathrm{~s}$ satisfy $c_{i}(t)=\Phi_{i} t$ for $t \in[0,1]$. Thus Eq. (25) reduce to:

$$
\left\{\begin{array}{l}
(1-t) \dot{K}^{e f f}=\sum_{i=1}^{\mathcal{N}}\left(K_{i}-K_{e f f}\right) \Phi_{i} P_{i} \\
(1-t) \dot{G}^{e f f}=\sum_{i=1}^{\mathcal{N}}\left(G_{i}-G_{e f f}\right) \Phi_{i} Q_{i}
\end{array} .\right.
$$

Last, the coefficients $P_{i}$ and $Q_{i}$ depend on the morphology of the inclusions; namely:

- For spherical inclusions in a 3-D matrix (Norris, 1985):

$$
\left\{\begin{array}{l}
P_{i}^{3 D}=\frac{K_{e f f}+K^{\star}}{K_{i}+K^{\star}} \\
Q_{i}^{3 D}=\frac{G_{e f f}+G^{\star}}{G_{i}+G^{\star}}
\end{array},\right.
$$

with $K^{\star}$ and $G^{\star}$ given by:

$$
\left\{\begin{array}{l}
K^{\star}=\frac{4}{3} G^{\star} \\
G^{\star}=\frac{G_{e f f}}{6} \frac{9 K_{e f f}+8 G_{e f f}}{K_{e f f}+2 G_{e f f}}
\end{array},\right.
$$

- For disk-shaped inclusions in a 2-D matrix (Thorpe and Sen, 1985):

$$
\left\{\begin{array}{l}
P_{i}^{2 D}=\frac{K_{e f f}+G_{e f f}}{K_{i}+G_{e f f}} \\
Q_{i}^{2 D}=\frac{2\left(K_{e f f}+G_{e f f}\right) G_{e f f}}{K_{e f f} G_{e f f}+\left(K_{e f f}+2 G_{e f f}\right) G_{i}}
\end{array} .\right.
$$


Using the differential scheme model thus defined, one can build a composite with an intrinsic variability in porosity, by considering as initial conditions the homogeneous solid response, $\left(K_{0}, G_{0}\right)=\left(K_{S}, G_{s}\right)$, and adding each phase as a solid-pore composite of porosity $\varphi_{i}=\varphi+t \delta_{\varphi}^{(i)}$, with bulk and shear moduli evaluated by the Mori-Tanaka scheme with porosity $\varphi_{i} \in$ $[0,1]$; that is $\left(K_{i}, G_{i}\right)=\left(K_{M T}\left(\varphi_{i}\right), G_{M T}\left(\varphi_{i}\right)\right)$ for the 2-D or 3-D configuration.

By way of example, consider (1) a 2-phase composite with $\varphi_{1}=\varphi-\Delta \varphi / \Phi_{1}$ and $\varphi_{2}=\varphi+\Delta \varphi / \Phi_{2}$, which satisfies $\left\langle\varphi_{t}\right\rangle=$ $\varphi$ and $\left\langle\varphi_{t}-\varphi\right\rangle=0$, while exhibiting a porosity standard deviation $\sigma_{\varphi}=\sqrt{\left\langle\left(\varphi_{t}-\varphi\right)^{2}\right\rangle}=(\Delta \varphi) \sqrt{\left(1 / \Phi_{1}+1 / \Phi_{2}\right)}$; and (2) a 4-phase composite with $\varphi_{1}=\varphi-2 \Delta \varphi / \Phi_{1}, \varphi_{2}=\varphi-\Delta \varphi / \Phi_{2}, \varphi_{3}=\varphi+\Delta \varphi / \Phi_{3}$ and $\varphi_{4}=\varphi+2 \Delta \varphi / \Phi_{4}$, satisfying $\left\langle\varphi_{t}\right\rangle=$ $\varphi,\left\langle\varphi_{t}-\varphi\right\rangle=0$, and $\sigma_{\varphi}=\Delta \varphi \sqrt{4 / \Phi_{1}+1 / \Phi_{2}+1 / \Phi_{3}+4 / \Phi_{4}}$. For these different configurations, the system of Eq. (26) is integrated numerically (Wolfram Research, Inc, 2016) from $t=0 \rightarrow 1$ to determine the effective Young's modulus for the 2-D and the 3-D system from:

$$
\left\{\begin{array}{l}
E_{e f f}^{2 D}=\frac{4 K_{e f f}(t=1) G_{e f f}(t=1)}{K_{e f f}(t=1)+G_{e f f}(t=1)} \\
E_{e f f}^{3 D}=\frac{9 K_{e f f}(t=1) G_{e f f}(t=1)}{3 K_{e f f}(t=1)+G_{e f f}(t=1)}
\end{array} .\right.
$$

Note that the condition $\varphi_{i} \in[0,1]$ limits the range of accessible average porosity $\varphi$ at a given $\sigma_{\varphi}$ value.

Fig. 5 displays sample output, $E_{\text {eff }} / E_{s}$, for the differential scheme model for a symmetrical 2-phase composite $\left(\Phi_{1}=\right.$ $\left.\Phi_{2}=1 / 2\right)$. A symmetrical 4-phase composite $\left(\Phi_{1}=\Phi_{2}=\Phi_{3}=\Phi_{4}=1 / 4\right)$ and an asymmetrical 2-phase solid $\left(\Phi_{1}=0.8\right.$ and $\Phi_{2}=0.2$ ) give the same results. Two points deserve attention: (i) at same $\sigma_{\varphi}$ value, all models provide the same effective stiffness vs. porosity response irrespective of the choice of symmetry and number of considered phases; (ii) the $E_{\text {eff }} / E_{s}$ response provided by the differential scheme model is strictly identical with the response of the asymptotic expansion model. This is most likely due to the Mori-Tanaka matrix-inclusion morphology assumed in both models.

\section{Results}

\subsection{Validation: elasticity of ordered systems}

The numerical LEM results are here validated for the ordered system against reference micromechanics solutions. This will permit us in the sequel to address the impact of disorder on elasticity with respect to the elasticity of the ordered systems, i.e. disorder-induced stiffness degradation.

In the simulations, ordered systems are referred to as the periodic arrangement of disk-shaped pores of radius $R$ or square-shaped pores of side-length $a$, in 2-D; and to their periodic 3-D analogs, i.e. spherical pores of radius $R$, or cubeshaped pores of side-length $a$. The porosity $\varphi$ of these systems is tuned by varying size $(R, a)$ and numbers $(N)$ of the pores in the simulation box of size $L$, and pore periodicity $l$. The critical porosity at percolation, $\varphi_{c},-$ that is the porosity above which the effective stiffness vanishes, is obtained for $R=l / 2$ for disks, $a=l$ for squares, $R=l / \sqrt{2}$ for spheres and $a=l$ for cubes:

$$
\left\{\begin{array}{rl}
\varphi_{c}^{\text {disk }} & =\frac{\pi}{4} \simeq 0.785 \\
\varphi_{c}^{\text {square }} & =1 \\
\varphi_{c}^{\text {sphere }} & =\frac{\pi}{12}(15-8 \sqrt{2}) \simeq 0.965 \\
\varphi_{c}^{\text {cube }} & =1
\end{array} .\right.
$$

Simulations were carried out by considering in the potential calibration different solid Poisson's ratios $\left(v_{s}=1 / 3\right.$ and $v_{s}=0.1$ in 2-D; $v_{s}=1 / 4$ and $v_{s}=0.1$ in 3-D). The LEM discretization was $n_{x}=n_{y}=221, n_{z}=2$, i.e. $n_{x} n_{y} n_{z}=97,682$ mass points in 2-D, and $n_{x}=n_{y}=n_{z}=61$, i.e. $n_{x} n_{y} n_{z}=226,981$ mass points in 3-D.

A large number of samples was generated by varying number $(N)$ and size $(R, a)$ of the pores $(N \in\{4,16,25,100,121$, $400,484\}$ in 2-D; $N \in\{8,27,64, \ldots 125,216,1000\}$ in 3-D). However, as Fig. 6 shows -in form of a plot of the dimensionless effective Young's modulus $E_{e f f} / E_{s}$ vs. porosity $\varphi$ - the ordered uniaxial elasticity response is insensitive to both number of pores and Poisson's ratio.

For the ordered system, the LEM simulation results show a fair amount of consistency with the continuum micromechanics solutions (Fig. 6). Specifically, at the low porosity limit $(\varphi \ll 1)$, the dilute approximations obtained from the 2-D and 3-D Mori-Tanaka solutions, Eqs. (17) and (18), compare fairly well with the simulation results, showing a linear decay 

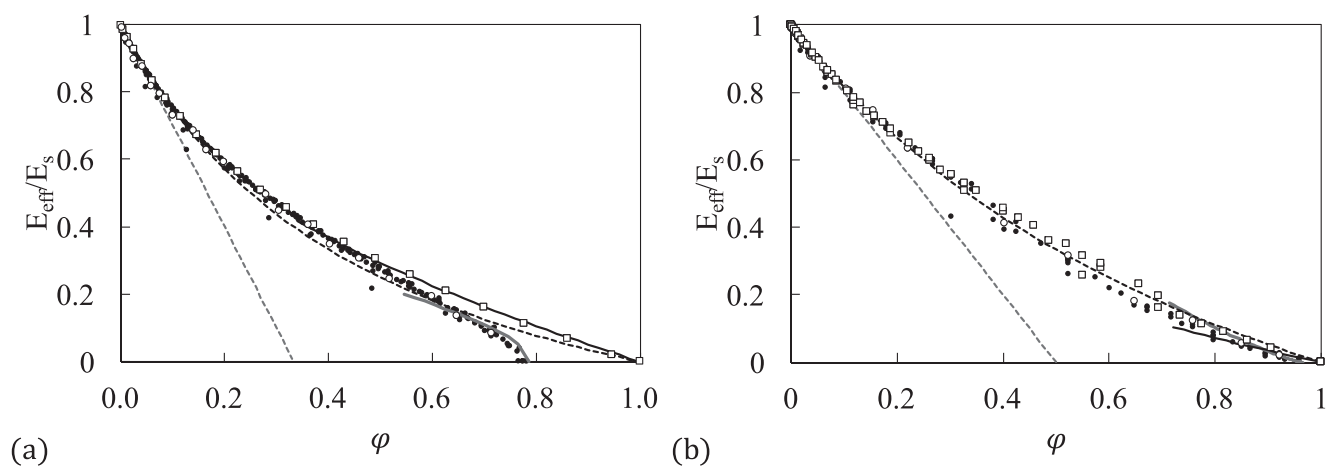

Fig. 6. Dimensionless effective Young's modulus: $E_{e f f}(\varphi) / E_{s}$ as function of the porosity. (a) 2 -D systems with disk-shaped pores $(\bullet: \nu=1 / 3$, $\circ$ : $v=0.1)$, square-shaped pores ( $\square$ ), dilute asymptot from Eq. (32) (gray broken line), Mori-Tanaka homogenization scheme from Eq. (17) (black broken line) and large porosity analytical solutions from Eqs. (37) (disks: gray solid line, squares: black solid line). (b) 3 -D systems with spherical pores $(\bullet: v=1 / 4$, $\circ$ : $v=0.1$ ), cubic pores ( $\square$ ), dilute asymptot from Eq. (33) (gray broken line), Mori-Tanaka homogenization scheme from Eq. (18) (black broken line) and large porosity analytical solutions from Eqs. (37) (spheres: gray solid line, cubes: black solid line).

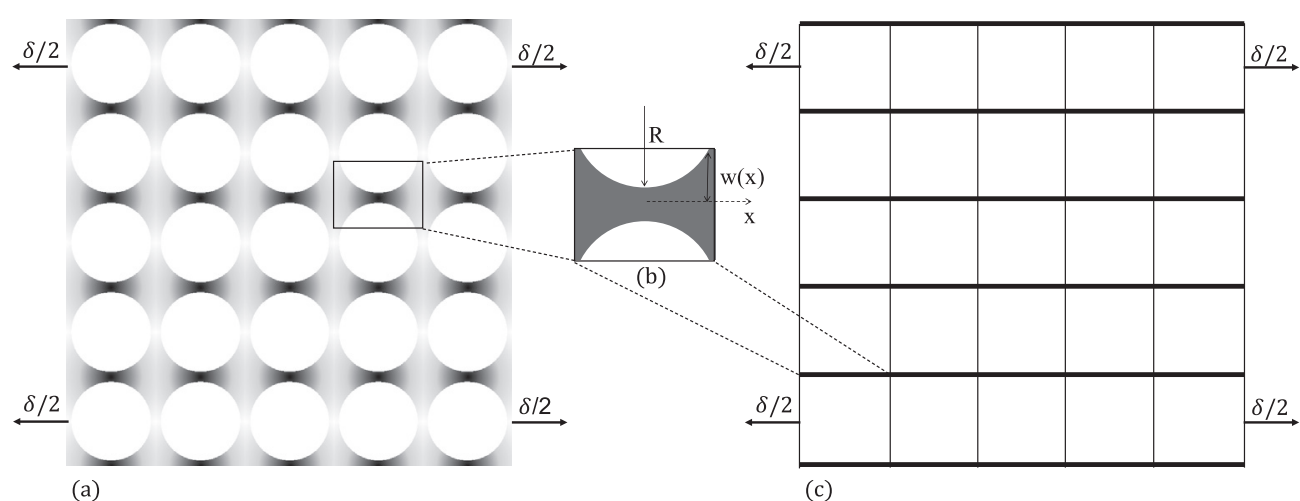

Fig. 7. Ordered system under uniaxial strain, (a) schematic stress map, the darker the higher the stress is, (b) zoom in between two neighboring pores and (c) equivalent geometry.

of $E_{\text {eff }} / E_{s}$ consistent with a Taylor development for $\varphi \ll 1$ (Day et al., 1992); that is ${ }^{3}$ :

$$
\left\{\begin{array}{l}
\frac{E_{M T}^{2 D}}{E_{S}} \simeq \frac{1-\varphi}{1+2 \varphi} \stackrel{\varphi \lll 1}{=} 1-3 \varphi+O\left(\varphi^{2}\right) \\
\frac{E_{M T}^{3 D}}{E_{S}} \simeq \frac{1-\varphi}{1+\varphi} \stackrel{\varphi \ll 1}{=} 1-2 \varphi+O\left(\varphi^{2}\right) .
\end{array}\right.
$$

These dilute approximations hold irrespective of the pore shape.

Beyond the dilute situation (Fig. 6), as mentioned by others (see e.g. Drach et al., 2016), we find that the Mori-Tanaka micromechanics model predicts reasonably well the stiffness vs. porosity behavior for a wide range of porosity; -except close to the percolation threshold for which the Mori-Tanaka scheme predicts $\varphi_{c}^{M T}=1$. The poor performance of the Mori-Tanaka scheme at high porosities is attributed to the stress concentration in narrow bands between the pores at high porosities (see Fig. 7 for the 2-D case with disk-shaped pores), that cannot be captured with mean-field averaging.

To test this hypothesis, the approach suggested by Day et al. (1992) is followed. Zones of stress concentrations are considered as a succession of infinitesimally small elastic beam elements of length $d x$ and section $S(x)$ which depends on the pore geometry. The approach is schematically sketched in Fig. 7. The beams follow a uniaxial stress-strain relation, $\sigma=E_{s} \varepsilon$, which is rewritten in terms of the infinitesimal displacement $d u=\varepsilon d x$ and the force $F=\sigma S(x)$ as $d u=F d x /\left(E_{S} S(x)\right)$. By integration one obtains $\Delta u=F / E_{S} \int d x / S(x)$. The effective constitutive relation in this inter-pore region reduces to $\sigma=F / S_{\text {eff }}=E_{e f f} \Delta u / l$ with $E_{\text {eff }}$ the effective stiffness and $S_{\text {eff }}$ the effective area of influence, i.e. $l d$ in $2-\mathrm{D}$ and $l^{2}$ in 3 -D. The effective stiffness in

\footnotetext{
${ }^{3}$ The approximation in Eq. (32) is valid for small values of $v_{s}$; whereas the approaximation in Eq. (33) is valid for $v \in[0,0.5]$, and is exact for $v=1 / 5$ and $v=1 / 3$.
} 
this high porosity limit is thus obtained from:

$$
\frac{E_{e f f}}{E_{s}}=\frac{F}{\left(E_{s} S_{e f f}\right)} \frac{l}{\Delta u}=\frac{l}{S_{e f f} \int(d x / S(x))},
$$

with the beam sections, $S(x)$, for the considered pore geometries, given by:

$$
\left\{\begin{array}{rl}
S^{\text {disk }}(x) & =\left(1-\frac{2 R}{l}+\frac{x^{2}}{R l}\right) l d=w(x) d \\
S^{\text {square }}(x) & =\left(1-\frac{a}{l}\right) l d \\
S^{\text {sphere }}(x) & =\left(1-\frac{\pi}{4} u^{2}(x)+s(x)\right) l^{2} \\
S^{\text {cube }}(x) & =\left(1-\frac{a}{l}\right) a l
\end{array},\right.
$$

where $u(x)=2 \sqrt{R^{2}-x^{2}} / l$, and $s(x)$ is the dimensionless function (see Appendix D):

$$
s(x)=\left\{\begin{array}{l}
0, \text { if } x \geq l / 2 \sqrt{u^{2}(x)-1} \\
u^{2}(x) \arctan \left(\sqrt{u^{2}(x)-1}\right)-\sqrt{u^{2}(x)-1}, \quad \text { otherwise. }
\end{array}\right.
$$

After integration, the high porosity limits around the percolation threshold $\left(\varphi=\varphi_{c}(1-\epsilon)\right.$ with $\left.\epsilon \ll 1\right)$ are obtained in the form $^{4}$ :

$$
\left\{\begin{array}{l}
\frac{E_{\text {eff }}^{\text {disk }}}{E_{s}} \simeq \frac{1}{\pi} \sqrt{\sqrt{\frac{\pi}{\varphi}}-\sqrt{\frac{\pi}{\varphi_{c}}}} \\
\frac{E_{\text {eff }}^{\text {square }}(\varphi)}{E_{s}} \simeq\left(\sqrt{\varphi_{c}}-\sqrt{\varphi}\right) \\
\frac{E_{\text {eff }}^{\text {cube }}(\varphi)}{E_{s}} \simeq\left(\sqrt[3]{\varphi_{c}}-\sqrt[3]{\varphi}\right)
\end{array} .\right.
$$

The comparison of the high-porosity limit expression (37) with simulation results in Fig. 6 thus provides evidence that the lack of accuracy of the Mori-Tanaka scheme close to the percolation threshold relates indeed to high stress concentrations that cannot be captured by zero-order averaging theories.

\subsection{Elasticity of disordered systems}

In contrast to the ordered systems with its fixed pore space configuration that entails one-to-one stiffness-porosity relation for a given pore shape, the effective stiffness of disordered systems is affected as well by the statistical distribution that characterizes the pore space configuration. Herein, the statistical ensemble generated for a given porosity is considered and the corresponding statistics of elastic moduli is evaluated with the focus on identifying a relevant disorder parameter able to capture the effect of disorder on the mean values of elastic moduli.

The effective stiffness-porosity $\left(E_{e f f} / E_{s}-\varphi\right)$ plots for a large number of randomly generated disordered systems in Fig. 8 confirm that disorder induces stiffness degradation compared to the ordered system. That is, at same porosity, the ordered system (Fig. 6) is an upper bound to the distribution of Young's modulus of disordered systems. This observation is consistent with its continuum micromechanics counterpart, for which it is well known that the Mori-Tanaka scheme representative of the ordered system- provides an upper-bound of stiffness (Dormieux et al., 2006; Hashin and Shtrikman, 1962). In the simulations, this upper bound is achieved by the periodic systems and approached by quasi-ordered systems ${ }^{5}$ with $\lambda=l /(2 R)$. At same porosity, the stiffness continuously decreases as $\lambda$ decreases, and reaches a lower bound for $\lambda \rightarrow$ 0 , corresponding to overlapping disks/spheres. A second important observation is that the percolation threshold decreases with disorder to approximately $\varphi_{c} \simeq 0.5$ in 2-D and $\varphi_{c} \simeq 0.8$ in 3-D. These simulation results are consistent with experimental results reported by Lobb and Forrester (1987) who studied randomly perforated square metal sheets where holes could possibly overlap (i.e. $\lambda \rightarrow 0$ ), and by Ishai and Cohen (1967) who studied cubic pores within a cubic epoxy matrix.

\footnotetext{
4 The case of spherical pores does not permit simple closed-form solutions, but requires numerical tools.

5 This is actually in perfect agreement with Hashin's theory (Hashin, 1962) in which a composite material is modeled by an assemblage of spheres containing one spherical pore and of porosity equal to the overall porosity. In our simulations, the upper bond corresponds to an assemblage of squares/cubes containing one circular/spherical pore and of porosity equal to the overall porosity.
} 

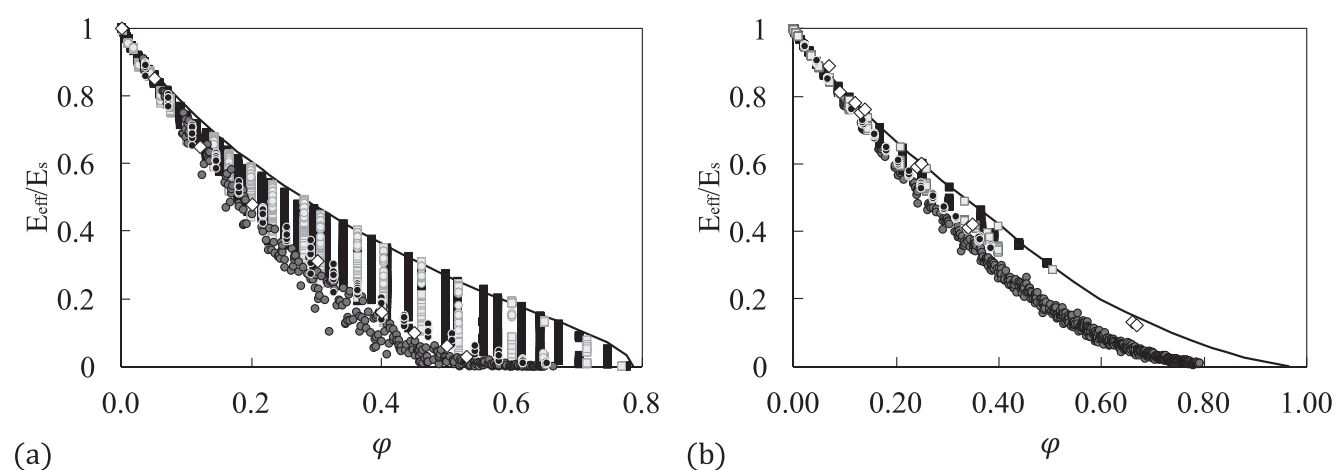

Fig. 8. Dimensionless effective Young's modulus: $E_{e f f}(\varphi) / E_{s}$ as function of the porosity. (a) 2-D samples, ordered structure: solid line, type 1 disorder with $N=100$ : light gray circles and type 2 disorder with $N=25$ : black squares, type 2 disorder with $N=100$ : light gray squares, type 3 disorder with $\lambda=1$ : black circles and type 3 disorder with $\lambda=0$ : dark gray disks, experimental data reported in Lobb and Forrester (1987): white diamonds. (b) 3-D samples, ordered structure: solid line, type 2 disorder with $N=27$ : black squares, type 2 disorder with $N=64$ : light gray squares, type 3 disorder with $\lambda=1$ : black circles and type 3 disorder with $\lambda=0$ : dark gray circles, experimental data reported in Ishai and Cohen (1967): white diamonds.

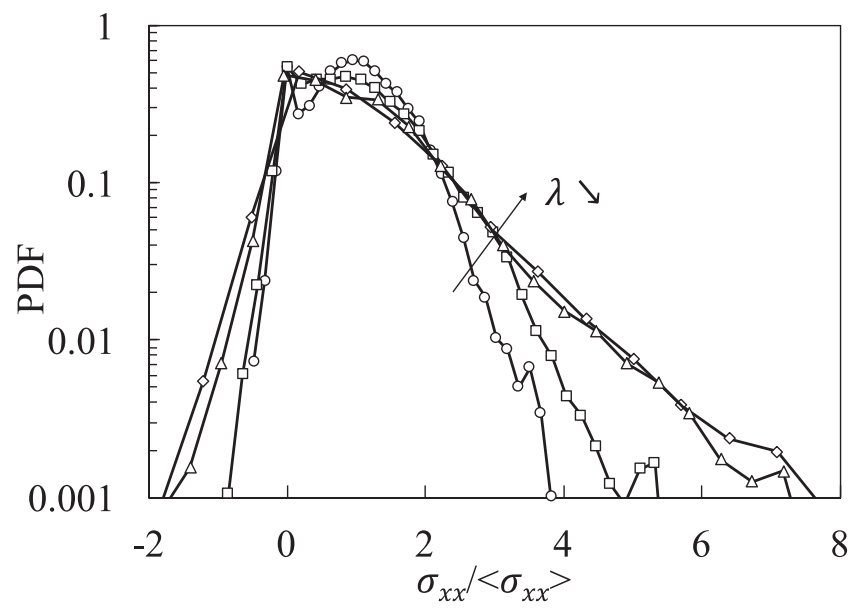

Fig. 9. Probability Density Function (PDF) of the normalized stress $\left(\sigma_{x x} /\left\langle\sigma_{x x}\right\rangle\right)$ in the solid phase for the same four systems as in Fig. 2 .

\section{Discussions}

The overall picture which thus emerges is that the (mean) porosity alone does not suffice as sole texture parameter to define the functional relation of the effective stiffness of disordered porous materials. We are thus in search of an additional relevant texture parameter, able to capture the disorder-induced stiffness degradation. When dealing with porous granular media, such a texture parameter could be a descriptor of the grain shape (see e.g. Fritsch et al., 2006; 2009; 2010; 2017; Sanahuja et al., 2010). For the materials studied here having a matrix/inclusion morphology and a uniform pore size and shape distribution (Fig. 1), another texture parameter is needed.

\subsection{Disorder parameter}

With this focus in mind, it is instructive to inspect the probability density function of the axial stress $\sigma_{x x}$-normalized by the average stress $\left\langle\sigma_{x x}\right\rangle$ in the solid phase- for different microstructures (Fig. 9). While the quasi-ordered system $(\lambda=$ $l /(2 R))$ exhibits an almost symmetric distribution around the mean value, the stress PDF broadens with increasing disorder $(l /(2 R) \geq \lambda \geq 0)$, with a significant skew on the tensile side. This skew is indicative of regions of high (tensile) stress concentrations (in the overall uniaxial tension test) around local pore clusters exhibiting higher local porosities than the average one, consistent with the local porosity distributions (see Fig. 3). This provides strong evidence that the disorderinduced stiffness degradation, at a fixed porosity, is attributable to stress concentrations due to pore clustering. This effect is more pronounced at high porosities compared to low porosities, leading to the observed shift in the percolation threshold.

It is thus natural to postulate that the clustering parameter $s_{a}$ introduced in Eq. (6) amplified by the mean porosity $\varphi$ could serve as an additional texture parameter to capture the impact of disorder on the effective elasticity. To test this hypothesis, the effective stiffness of the disordered systems is first corrected by the effective stiffness of the ordered system 

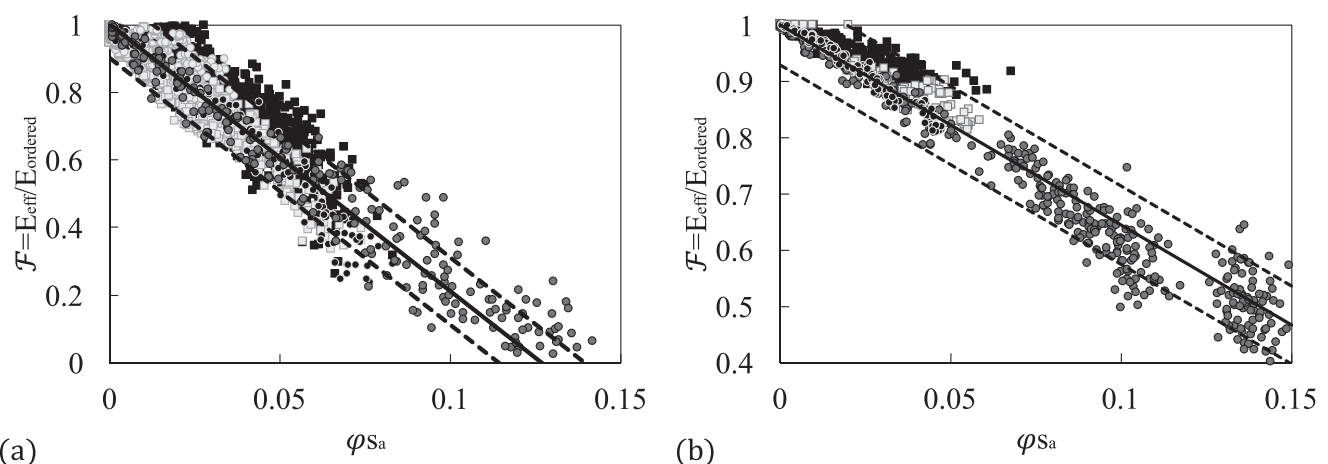

Fig. 10. Dimensionless disordered Young's modulus: $\mathcal{F}\left(\varphi s_{a}\right)=E_{e f f}\left(\varphi, s_{a}\right) / E_{\text {ordered }}(\varphi)$ as function of the disorder parameter $s_{a} \varphi$. (a) 2-D samples simulation results: type 1 disorder $(N=100)$ : light gray circles, type 2 disorder with $N=25$ : black squares, type 2 disorder with $N=100$ : light gray squares, type 3 disorder with $\lambda=1$ : black circles and type 3 disorder with $\lambda=0$ : dark gray circles, up to $\varphi=\varphi_{c}^{2 D}$. (b) 3 -D samples simulation results, type 2 disorder with $N=27$ : black squares, type 2 disorder with $N=64$ : light gray squares, type 3 disorder with $\lambda=1$ : black circles and type 3 disorder with $\lambda=0$ : dark gray circles, up to $\varphi=\varphi_{c}^{3 D}$. Black lines correspond to relation (39).

at same mean porosity (shown in Fig. 6); to arrive at the dimensionless form:

$$
\frac{E_{e f f}\left(\varphi, s_{a}\right)}{E_{\text {ordered }}(\varphi)}=\mathcal{F}\left(\varphi s_{a}\right)
$$

This dimensionless form is displayed in Fig. 10 and shows an almost linear decrease of the normalized effective stiffness with the disorder parameter, $\varphi s_{a}$. More specifically, for small values of $\varphi s_{a}$, the variability in the effective stiffness is low. However, as the clustering $s_{a}$ increases, the effective stiffness of a given system can become much smaller than the stiffness of the ordered system of same porosity. The results thus obtained from extensive numerical simulations support the conjecture that the clustering index $\left(s_{a}\right)$ weighted by the mean porosity $(\varphi)$ is an appropriate texture parameter $\left(\varphi s_{a}\right)$ to describe the disorder-induced stiffness degradation of porous materials. A fit of the data in Fig. 10 suggests the following form:

$$
\mathcal{F}\left(\varphi s_{a}\right)=1-k \times \varphi s_{a} \pm \Delta / 2,
$$

with fitted values $k^{2 D} \simeq 7.9, \Delta^{2 D} \simeq 0.2$ in 2-D (Fig. 10(a)), and $k^{3 D} \simeq 3.6, \Delta^{3 D} \simeq 0.14$ in 3-D (Fig. 10(b)) which guarantee a $99 \%$ confidence on the predicted value.

This functional relation (39) also permits the identification of the percolation threshold from:

$$
\mathcal{F}\left(\varphi_{c} s_{a}\right) \rightarrow 0 \Leftrightarrow \varphi_{c} \simeq 1 /\left(k s_{a}\right) .
$$

Using the maximum value for $s_{a}$ from simulations, namely $s_{a}=0.27$ for overlapping disks, the estimated 2-D percolation threshold is $\varphi_{c}^{2 D}=1 / 7.9 / 0.27=0.47$, which compares fairly well with the one obtained from Fig. $8, \varphi_{c}^{2 D} \simeq 0.5$. Finally, it is worth-noting that the extent of fluctuations for all values of $\varphi s_{a}$ is almost constant (captured by $\Delta$ in fitting relation (39)). This suggests that the small fluctuations in Fig. 10 around the linear decrease are attributable to the intrinsic fluctuations of the distributions used for the generation of pore configurations.

\subsection{Limits of continuum micromechanics modeling}

We are now left with comparing the simulation results with the micromechanics solutions introduced in Section 2.3. To do so, one needs to define a clustering index $s_{a}$ for the micromechanics models, which rely on the same three assumptions:

1. The porous material modeled consists of two phases of porosity $\varphi_{i}$ and volume fraction $\Phi_{i}(i \in\{1,2\})$,

2. The spatial distribution of each phase in the volume corresponds to a Mori-Tanaka morphology,

3. Each phase responds as a Mori-Tanaka porous solid $\left(E_{i}=E_{M T}\left(\varphi_{i}\right)\right)$.

The two-phase solids depicted in Fig. 11 satisfy these three assumptions. They consist of a periodic arrangement (periodicity $2 l$ ) of disks of two different radii, $R_{1}$ and $R_{2}$, such that $\varphi_{i}=\pi\left(R_{i} / l\right)^{2}$. Each phase thus corresponds to a Mori-Tanaka composite for porosities not too close to the percolation threshold $\varphi_{c}$, and the spatial arrangement of the two-phases is ordered as well, reminiscent of a Mori-Tanaka morphology. The average porosity of the considered systems is $\varphi=\left(\varphi_{1}+\varphi_{2}\right) / 2$, and the standard deviation of the local porosity as defined by Eq. (15) is $\sigma_{\varphi}^{2}=\left(\left(\varphi_{1}-\varphi\right)^{2}+\left(\varphi_{2}-\varphi\right)^{2}\right) / 2$.

For these systems, we introduce the clustering index, $s_{a}$, as the standard deviation of the porosity $\varphi_{a}$ measured in a square observation window of side-length $a$. Akin to the simulations, the size of the observation window is chosen such that $s_{a}=0$ for uniform systems $\left(R_{1}=R_{2}=\sqrt{\varphi / \pi} l\right)$, so that $a=l$. Porosity fluctuations are introduced by considering different pore radii $\left(R_{1} \neq R_{2}, \sigma_{\varphi} \neq 0\right)$ resulting in a non-uniform local porosity distribution, $\varphi_{a}$, which varies between $\varphi_{1}$ and $\varphi_{2}$. 


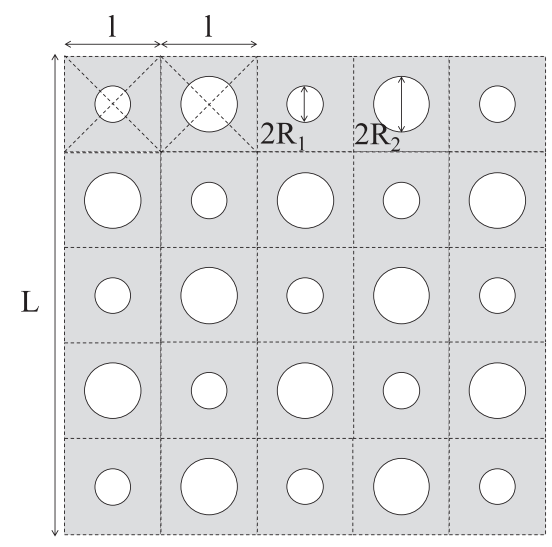

Fig. 11. Two-phase periodic porous solid.

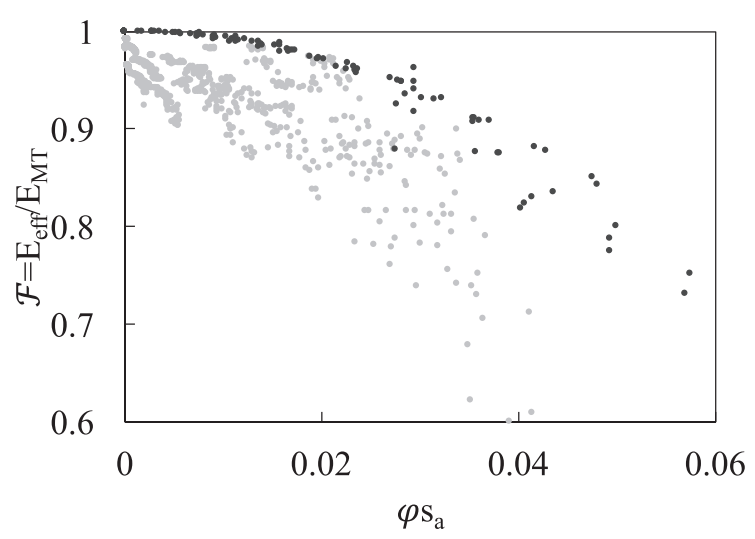

Fig. 12. Dimensionless disordered Young's modulus: $\mathcal{F}_{M T}\left(\varphi s_{a}\right)=E_{e f f}\left(\varphi, s_{a}\right) / E_{M T}(\varphi)$ as function of the disorder parameter $s_{a} \varphi$. Dark gray disks correspond to the micromechanics solution while light disks correspond to type 1 disorder with $N=100$.

The 2-D-clustering index (6) of the micromechanics model is obtained from:

$$
s_{a}\left(\varphi_{1}, \varphi_{2}\right)=\frac{1}{(2 l)^{2}} \int_{x=-l}^{l} \int_{y=-l}^{l}\left(\varphi_{a}(x, y)-\varphi\right)^{2} d y d x,
$$

where $\varphi_{a}(x, y)$ is the porosity measured in an observation window centered at the point of coordinates ( $\left.x, y\right)$. Details for the calculation of $\varphi_{a}(x, y)$ are given in Appendix E.

In order to compare the simulation results to the micromechanics model, the effective stiffness predicted by the smallfluctuation model (i.e. Eq. (24)) and by the 2-phase differential scheme model (i.e. Eq. (30)) are normalized by the effective stiffness of the ordered systems as predicted by the Mori-Tanaka scheme (i.e., Eq. (17)), in the form:

$$
\frac{E_{e f f}\left(\varphi, s_{a}\right)}{E_{e f f}\left(\varphi, s_{a}=0\right)}=\frac{E_{e f f}\left(\varphi, s_{a}\right)}{E_{M T}(\varphi)}=\mathcal{F}_{M T}\left(\varphi s_{a}\right) \text {. }
$$

This comparison is shown in Fig. 12 for different mean porosities $\varphi=\left(\varphi_{1}+\varphi_{2}\right) / 2$, and different clustering index values $s_{a}\left(\varphi_{1}, \varphi_{2}\right)$, spanning the relevant range of the disorder parameter, $\varphi s_{a}$, covered by the simulation results. While the micromechanics models qualitatively predict a disorder-induced stiffness degradation compared to the ordered system, they somewhat underestimate this effect compared to simulations. In the case of the Mori-Tanaka-based asymptotic expansion model (Eq. (24)), one could argue that the model's focus is on small porosity fluctuations $\left(t \ll 1\right.$ or $\left.\sigma_{\varphi} \ll 1\right)$, for which reason it may underestimate the stiffness degradation for larger values of porosity fluctuations which characterize the simulation samples (see Fig. 3). In its turn, the underestimation of the differential scheme may be attributed to the ergodicity assumption that underlines the iterative construction process, since -as (Norris, 1985) noted- "at each stage [of the iterative construction process] the material is assumed to be homogeneous" . This assumption of iterative homogeneity cannot capture the impact of localized stress concentrations around porosity clusters (see Fig. 9) that entail the pronounced linear decrease of the stiffness with $\varphi s_{a}$. One should also keep in mind that the $s_{a}$ value (41) is exact only for systems such as the one depicted on Fig. 11. 


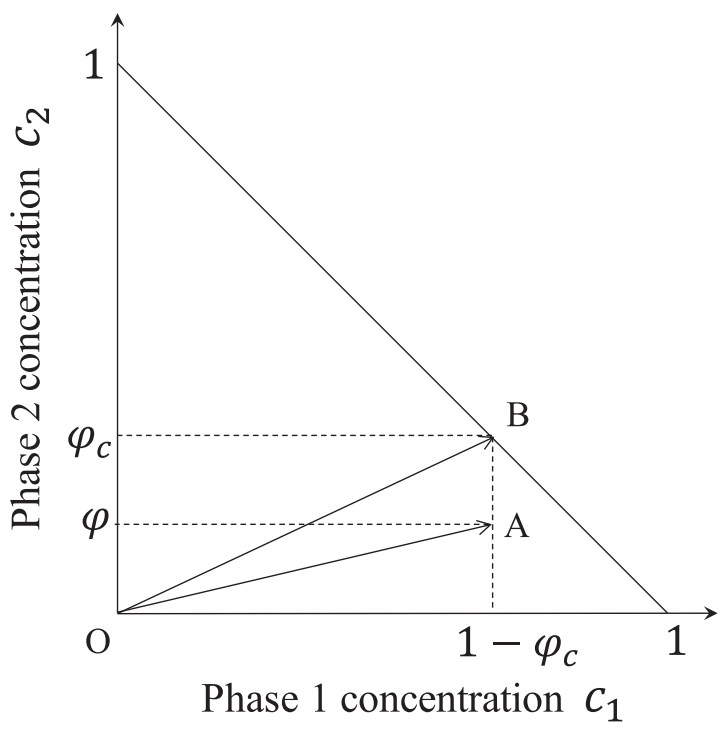

Fig. 13. Integration path for the lower bound differential scheme. Path $O A$ ( $O$ corresponding to $t=0$ and $A$ corresponding to $t=1$ ) is used for intermediate values of the porosity $\left(0<\varphi<\varphi_{c}\right)$ and path $O B$ ( $O$ corresponding to $t=0$ and $B$ corresponding to $\left.t=1\right)$ is used for the porosity at percolation $\varphi=\varphi_{c}$.

All this supports the idea that the elasticity of disordered porous systems requires an explicit role for a texture parameter encapsulated here in the disorder parameter $\varphi s_{a}$.

There is one exception, namely when disorder is statistically confined to a single disorder configuration, e.g. by a fixed value of the degree of impenetrability, $\lambda=R_{a p p} / R$. For 2-D-systems, Markov et al. (2012) observed, for $\lambda=0$, corresponding to the overlapping disk model, that a specific application of the differential scheme (i.e. Eq. (25)) is able to capture the disorder-induced stiffness degradation. The particular differential scheme model is a two-phase system with one phase representing the solid phase $\left(K_{0}=K_{1}=K_{s}\right.$ and $\left.G_{0}=G_{1}=G_{s}\right)$, the other the pore phase of zero-elasticity $\left(K_{2}=0, G_{2}=0\right)$. The concentrations are given in the form:

$$
\left\{\begin{array}{l}
c_{1}(t)=\left(1-\varphi_{c}\right) t \\
c_{2}(t)=\varphi t \\
c_{0}(t)=1-c_{1}(t)-c_{2}(t)
\end{array}, t \in[0,1]\right.
$$

where $\varphi_{c}$ is the percolation threshold observed in experiments and/or simulations ${ }^{6}$. Indeed, an integration of Eq. (25) from $t=0$ to 1 along the path shown in Fig. 13, for porosity values in the range $0 \leq \varphi \leq \varphi_{c}$, provides a functional relation for the effective stiffness $E_{\text {eff }}$ in function of porosity $\varphi$. Normalized by stiffness $E_{s}$ of the solid, the excellent agreement of this model with simulation results in both 2-D and 3-D (Fig. 14) confirms that if the percolation threshold is known, the described differential scheme is indeed able to reproduce the stiffness vs. porosity behavior of this particular porous material system of well-defined disorder characteristic. In the simulations, the results obtained on samples generated by GCMC with fully penetrable pore disks (2-D) or spheres (3-D), for which $\lambda=0$, define a lower bound of the effective stiffness $E_{e f f}(\varphi, \lambda=0) / E_{\text {ordered }}(\varphi)$. Interestingly, a similar stiffness vs. porosity behavior can be captured by a self-consistent formalism applied to elongated crystals (Sanahuja et al., 2010). This suggests that in the fully disordered samples (Fig. 2(d)), stresses concentrate in narrow regions that may be correctly modeled by an assembly of elongated grains. For further analogy between porous systems and granular media, the reader is directed to Laubie et al. (2017b).

One could speculate that the good agreement of this micromechanics model with the simulation results comes from fixing the type of disorder in the micromechanics model through the combination of (1) the model choice of what Lacey (1954) described as 'completely unmixed material' with fixed porosity values for the two phases (i.e. $\varphi_{1}=0$ and $\varphi_{2}=1$ ), and (2) by fixing -as input- the percolation threshold in Eq. (43) representative of this disorder-induced stiffness degradation of samples generated with $\lambda=0$. There is thus no reason that the model could predict anything but the lower bound of the stress-induced stiffness degradation as a function of the porosity, for which it is designed, unless an additional texture or length scale parameter is introduced to enrich the poor statistical description of the 'completely unmixed material'. To illustrate our purpose, we here seek for a characteristic functional relation between the clustering index $s_{a}$ and the porosity

\footnotetext{
${ }^{6}$ When the value $\varphi_{c}=1$ is chosen, this modified version of the differential scheme degenerates to the classical model giving $E_{d i f f}^{2 D} / E_{s}=(1-\varphi)^{3}$ and $E_{\text {diff }}^{3 D} / E_{s}=(1-\varphi)^{2}$ in 2-D and 3-D, respectively.
} 

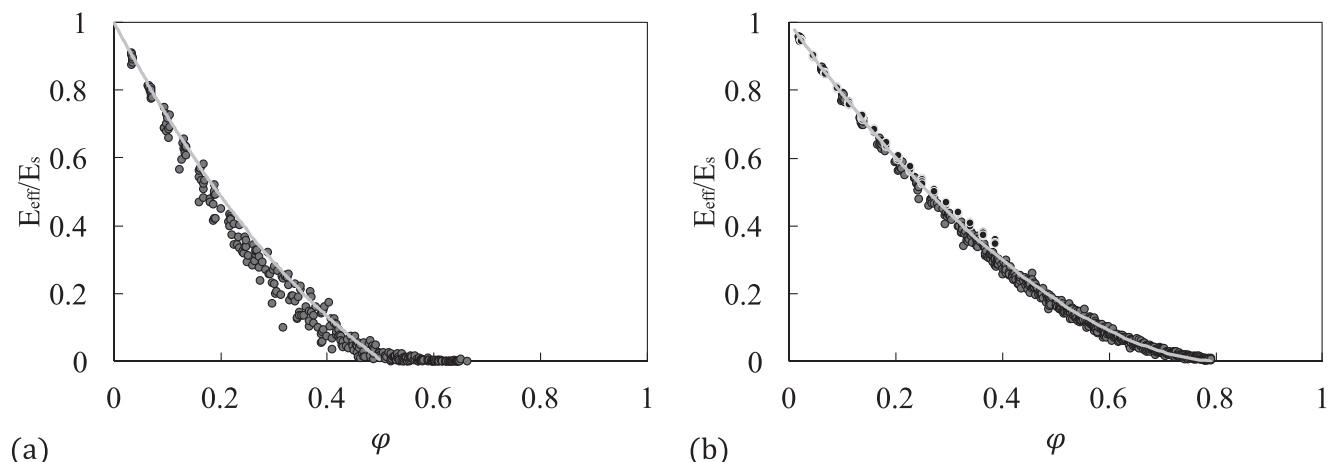

Fig. 14. Dimensionless effective Young's modulus: $E_{\text {eff }}(\varphi) / E_{s}$ as function of porosity the. Solid line: lower bound differential scheme in 2 -D with $\varphi_{c}=0.5$ (a) and in 3-D with $\varphi_{c}=0.8$ (b). Symbols: type 3 disorder with $\lambda=1$ (black disks) and type 3 disorder with $\lambda=0$ (dark gray disks).
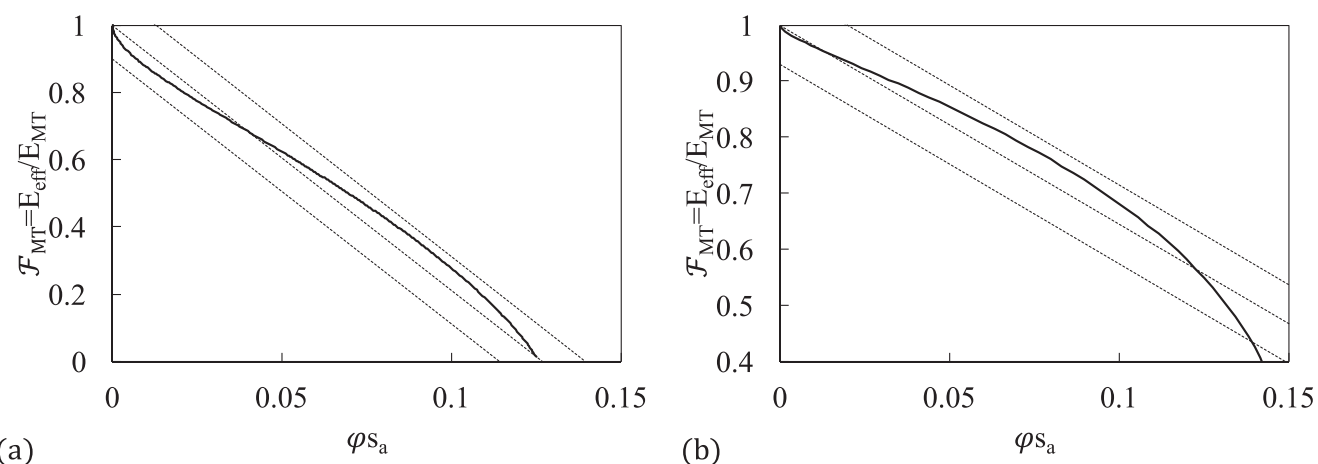

Fig. 15. Dimensionless disordered Young's modulus: $\mathcal{F}_{M T}\left(\varphi s_{a}\right)=E_{e f f}\left(\varphi, s_{a}\right) / E_{M T}(\varphi)$ as function of the disorder parameter $s_{a} \varphi$. Solid line: lower bound differential scheme in 2-D with $\varphi_{c}=0.5$ (a) and in 3-D with $\varphi_{c}=0.8$ (b) where $s_{a}=s_{a}^{\text {diff }}$ (Eq. (44)). Dashed lines correspond to the envelop defined by Eq. (39).

$\varphi$, calibrated against the simulation results. That is, from Fig. B.16 (see Appendix B):

$$
s_{a}^{\text {diff }} \simeq \varphi(1-\varphi) .
$$

A fairly consistent prediction of the disorder-induced stiffness degradation (38) in function of the disorder parameter is obtained when using the differential scheme model (Eqs. (43)) and the disorder parameter expression (Eq. (44)) in $E_{e f f}^{\text {diff }}\left(\varphi, s_{a}^{\text {diff }}\right) / E_{M T}(\varphi)$. This holds true for both 2-D and 3-D systems (Fig. 15). It should, however, be noted that this predictive quality comes at the price of two additional input information, namely the disorder-induced percolation threshold $\left(\varphi_{c}\right)$ and the link between the clustering index and the porosity (Eq. (44)).

\section{Conclusions}

We have shown that disorder plays a critical role on the effective elasticity of porous materials. In addition to the sample porosity, the consideration of an appropriate 'disorder parameter' is required to capture this microstructural effect and to predict the disorder-induced stiffness degradation of inhomogeneous porous solids. By disorder-induced stiffness degradation, we mean that the effective stiffness of disordered porous samples (Fig. 1(c)) is lower than the stiffness of the ordered porous material (Fig. 1(a)) of same (mean) porosity. We come to this conclusion from an investigation of the elastic behavior of a large range of 2-D and 3-D porous samples randomly generated with Monte Carlo-type algorithms, that exhibit, at same porosity, very different microstructures as quantified in terms of 2-point probability functions (Fig. 2) and local porosity distributions (Fig. 3). The following points deserve attention:

1. The disorder-induced stiffness degradation of porous materials originates from stress concentrations around pore clusters. This effect is more pronounced at high porosities than at low porosities, leading to a shift in the percolation threshold compared to the ordered system.

2. This effect of pore clustering on the effective stiffness of disordered porous materials is well captured -in first orderby means of a weighted clustering index, the disorder parameter, $\varphi s_{a}$. The clustering index, $s_{a}$, is a measure of the standard deviation of the local porosity throughout the sample volume. Multiplied by the mean porosity, an (almost) 
linear decrease of the disorder-induced stiffness degradation with $\varphi s_{a}$ is found, for all samples in both 2-D and 3-D. This disorder parameter, which only requires porosity distribution measurements, provides a means to characterize the disorder-induced stiffness degradation of disordered porous materials.

3. The lack of such disorder characterization in classical continuum micromechanics-based models based on spherical inclusions, which is equivalent to the absence of a length scale characterizing the stress concentrations in disordered porous materials, limits the predictive capabilities of such models to capture disorder-induced stiffness degradation quantitatively. This was illustrated through the application of two micromechanics models that permit an account of the variability in porosity distribution; namely the small-fluctuation model by Gărăjeu and Suquet (2007) (which was generalized to 3-D- and 2-D-compressible elastic solids), and the differential scheme model adapted from Norris (1985) to account for porosity variations.

4. Such continuum-based micromechanics model cannot quantitatively account for disorder-induced stiffness degradation unless additional information is provided that overcomes their overriding homogeneity assumption. This was illustrated through an extension of Markov et al. (2012)'s 2-phase differential scheme model to 'completely unmixed porous materials' in both 2-D and 3-D, which defines a lower bound of the disorder-induced stiffness degradation. The quantitative capabilities of such models may be improved through the consideration of texture-related quantities, namely the percolation threshold $\varphi_{c}$ and a link between the clustering index and the porosity.

5. The present study focused on only one type of disorder attributed to the random spatial distribution of spherical pores in a linear elastic solid matrix. A natural extension of this work would consist in putting to test the ability of the disorder parameter introduced here to characterize other types of disordered heterogeneous solids such as systems with solid inclusions or polydisperse systems.

\section{Acknowledgments}

Research carried out within the X-Shale Hub@MIT, the Science and Engineering of Gas Shale, a collaboration between Shell, Schlumberger and MIT, enabled through MIT's Energy Initiative. FR would like to acknowledge the support of the ICoME2 Labex (ANR-11-LABX- 0053) and the A*MIDEX projects (ANR-11-IDEX-0001-02) cofunded by the French program Investissements d'Avenir, managed by the French National Research Agency (ANR). The authors acknowledge Vincent Richefeu (Université Joseph Fourier) and Jean-Yves Delenne (Montpellier SupAgro) who provided the backbone of the LEM code used for the simulations.

\section{Appendix A. Porosity of a cubic box with a single spherical pore}

The porosity of a cubic box of side $l$ containing a sphere of radius $R$ is $\varphi=4 / 3 \pi(R / l)^{3}$ for $R \leq l / 2$. When $R>l / 2$, that is when the spherical pore intersects the sides of the cube, the porosity becomes $\varphi=\left(4 / 3 \pi R^{3}-6 v_{\text {cap }}(R)\right) / l^{3}$ where the volume $v_{\text {cap }}(R)=v(R) l^{3}$ of the 6 spherical caps getting off the cube have been subtracted from the sphere volume $4 / 3 \pi R^{3}$. This volume is:

$$
\begin{aligned}
& v_{\text {cap }}(R)=\int_{z=0}^{R-l / 2} \pi r^{2}(z) d z \\
& =\int_{z=0}^{R-l / 2} \pi\left[R^{2}-\left(\frac{l}{2}-z\right)^{2}\right] d z \\
& =\frac{\pi}{24}\left(\frac{2 R}{l}-1\right)^{2}\left(\frac{4 R}{l}+1\right) l^{3} .
\end{aligned}
$$

\section{Appendix B. Clustering index for penetrable two-dimensional disks}

The clustering index $s_{a}$ is a measure of the variability (that is the fluctuation) in the local porosity. This local porosity $\varphi_{a}$ is measured in an observation window ${ }^{7}$ of area $V_{0} \sim a^{2}$. The statistical average of the local porosity is the total porosity, $\varphi=\left\langle\varphi_{a}\right\rangle$. As of the clustering index, it is defined by Eq. (6).

If the observation window is very small $(a \rightarrow 0)$, the measured local porosity is either 0 if the point of measure is in the solid phase (with probability $1-\varphi$ ) or 1 if the point of measure is in the solid phase (with probability $\varphi$ ). The clustering index is thus:

$$
\lim _{a \rightarrow 0} s_{a}=\sqrt{(1-\varphi)(0-\varphi)^{2}+\varphi(1-\varphi)^{2}}=\sqrt{\varphi(1-\varphi)} .
$$

\footnotetext{
7 The theoretical developments (Bayer, 1964; Lu and Torquato, 1990) presented here consider a disk-shaped observation window (radius a) while in the numerical simulations presented in this paper, this observation window is square-shaped (side-length $a$ ).
} 

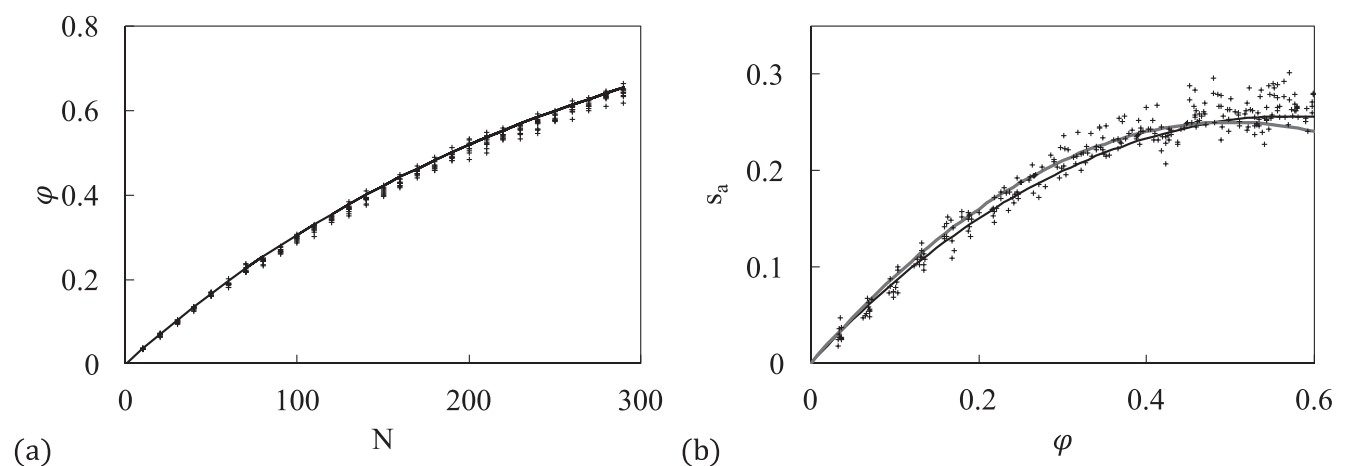

(a)

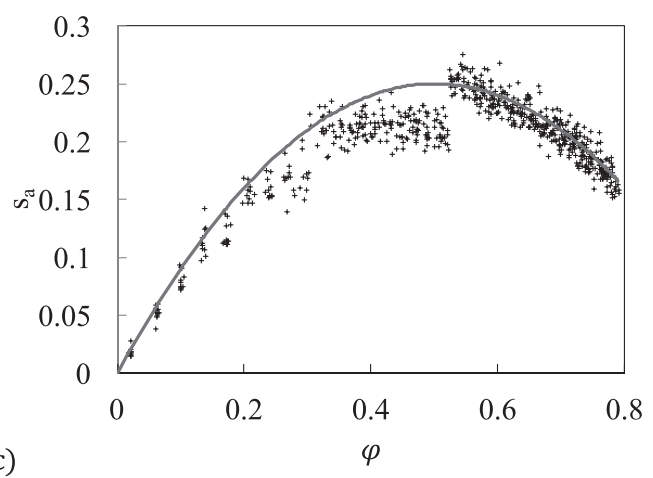

Fig. B.16. (a) Porosity as a function of the number of pores. (b) and (c) 2D- and 3D-clustering index as a function of porosity, respectively. Crosses correspond to simulation results $(R / L \simeq 0.04$ ). Black lines correspond in (a) and (b) to Eqs. (B.3) and (B.7), respectively. Gray lines in (b) and (c) correspond to approximation (B.8).

In the other limit case, $a \rightarrow L$ (where $L$ is the size of the studied system), the local porosity does not exhibit any variability, resulting in a clustering index:

$$
\lim _{a \rightarrow L} s_{a}=0 .
$$

Consider a random system of fully penetrable $(\lambda=0)$ disks of radius $R$ and area $V_{1}=\pi R^{2}$ filling a square box of area $V=L^{2}$. For such a system, the one-point and two-point probability functions of the solid phase (Eqs. (4) and (3), using the characteristic function of the solid phase instead of the one of the pore phase) are given by (see e.g. Lu and Torquato, 1990; Torquato and Beasley, 1986):

$$
\left\{\begin{array}{l}
S_{1}^{\text {solid }}=\exp \left\{-\frac{N V_{1}}{V}\right\}=1-\varphi \\
S_{2}^{\text {solid }}(r)=\exp \left\{-\frac{N V_{1}}{V}\left(2-g\left(\frac{r}{2 R}\right)\right)\right\}
\end{array}\right.
$$

with $N / V$ the number of disks per unit area and $V_{1} \times(2-g(r /(2 R)))$ the union area of two disks of radius $R$ at a distance $r$. Function $g$ is given by:

$$
g(u)=\frac{2}{\pi}\left(\arccos u-u \sqrt{1-u^{2}}\right) H(1-u)
$$

with $H(x)$ the Heaviside function. Bayer (1964) first derived the exact relation between the clustering index and the twopoint probability function in the 2-D case while Lu and Torquato (1990) gave a general relation in $D$-dimension. The clustering index for the system studied here is (Bayer, 1964):

$$
s_{a}^{\max }=\exp \left\{-\frac{N V_{1}}{V}\right\} \sqrt{\frac{V_{1}}{V_{0}} \int_{u=0}^{1}\left(\exp \left\{\frac{N V_{1}}{V} g(u)\right\}-1\right) 8 u g\left(u \sqrt{\frac{V_{1}}{V_{0}}}\right) d u . ~}
$$

In the simulations, the choice of the size of the observation window was such that $s_{a}=0$ for the reference (ordered) systems: $\varphi=\pi R^{2} / a^{2}$, i.e. $V_{1} / V_{0}=\varphi$. Injecting this relation and (B.3) in (B.6) gives a one-to-one relation between the clustering 
index and the porosity:

$$
s_{a}^{\max }(\varphi)=(1-\varphi) \sqrt{\varphi \int_{u=0}^{1}\left((1-\varphi)^{-g(u)}-1\right) 8 u g(u \sqrt{\varphi}) d u} .
$$

Fig. B.16 compares simulation data to the theoretical predictions for the porosity vs. number of pores behavior (Eq. (B.3)) and the clustering index vs. porosity behavior (Eq. (B.7)) and shows an excellent agreement between the two. In addition, it is shown that (Eq. (B.7)) can be approximated by:

$$
s_{a}^{a p p}(\varphi)=\varphi(1-\varphi) .
$$

This approximation is also valid for 3-D systems (Fig. B.16(c)).

We can here make an analogy with the configuration entropy used in statistical mechanics. This entropy relates to the number of ways a system of particles can arrange itself. For two-state systems (0 or 1), the configuration entropy $S$ is: $S=$ $-P \ln P-(1-P) \ln (1-P)$ with $P$ the probability of being in state 1 . In the maximum disorder limit $(P=1 / 2)$, the entropy reaches its maximum value while it vanishes for $P=0$ or 1 .

\section{Appendix C. Micromechanics solution for porous materials with small porosity variations in a compressible isotropic matrix}

Gărăjeu and Suquet (2007)'s results are here generalized to the case of spherical pores in a compressible isotropic matrix for which the stiffness tensor is (see e.g. Dormieux et al., 2006):

$$
\mathbf{C}^{3 D, M T}\left(\varphi_{0}\right)=3 K_{M T}\left(\varphi_{0}\right) \mathbf{J}+2 G_{M T}\left(\varphi_{0}\right) \mathbf{K}
$$

where $\mathbf{J}=1 / 3(\mathbf{i} \otimes \mathbf{i})$ is the projector over spherical tensors and $\mathbf{K}=\mathbf{I}-\mathbf{J}$ is the projector over deviatoric tensors (i is the second-order identity tensor while $\mathbf{I}$ is the fourth-order identity tensor). The bulk and shear moduli, $K_{M T}$ and $G_{M T}$, are:

$$
\left\{\begin{array}{l}
K_{M T}\left(\varphi_{0}\right)=\frac{K_{s}\left(1-\varphi_{0}\right)\left(1-\alpha_{0}\right)}{1-\alpha_{0}(1-\varphi)} \\
G_{M T}\left(\varphi_{0}\right)=\frac{G_{s}\left(1-\varphi_{0}\right)\left(1-\beta_{0}\right)}{1-\beta_{0}(1-\varphi)}
\end{array}\right.
$$

with:

$$
\left\{\begin{array}{l}
\alpha_{0}=\frac{3 K_{s}}{3 K_{s}+4 G_{s}} \\
\beta_{0}=\frac{6\left(K_{s}+2 G_{s}\right)}{5\left(3 K_{s}+4 G_{s}\right)}
\end{array} .\right.
$$

In this context, the elastic energy is:

$$
\tilde{w}\left(\varphi_{0}, \boldsymbol{\epsilon}\right)=\frac{9}{2} \lambda_{M T}\left(\varphi_{0}\right) \epsilon_{m}^{2}+\frac{3}{2} G_{M T}\left(\varphi_{0}\right) \epsilon_{e q}^{2}
$$

with $\lambda_{M T}\left(\varphi_{0}\right)=K_{M T}\left(\varphi_{0}\right)-2 / 3 G_{M T}\left(\varphi_{0}\right)$ the first Lamé parameter, $\epsilon_{e q}=\sqrt{2 / 3(\boldsymbol{\epsilon}: \boldsymbol{\epsilon})}$ the equivalent strain and $3 \epsilon_{m}=\operatorname{tr}(\boldsymbol{\epsilon})$ the volume strain.

Introduce the polarization field $\tau$ defined as:

$$
\begin{aligned}
\boldsymbol{\tau} & =\frac{\partial^{2} \tilde{\boldsymbol{w}}}{\partial \varphi \partial \boldsymbol{\epsilon}}\left(\varphi_{0}, \overline{\boldsymbol{\epsilon}}\right) \\
& =\tau_{m} \mathbf{i}+\frac{2}{3} \tau_{e q} \overline{\mathbf{e}}
\end{aligned}
$$

with $\overline{\mathbf{e}}=\left(\overline{\boldsymbol{\epsilon}}-\epsilon_{m} \mathbf{i}\right) / \epsilon_{e q}$ and:

$$
\left\{\begin{array}{l}
\tau_{m}=\frac{1}{3} \frac{\partial^{2} \tilde{w}}{\partial \varphi \partial \epsilon_{m}}\left(\varphi_{0}, \overline{\boldsymbol{\epsilon}}\right)=3 \epsilon_{m} \frac{\partial \lambda_{M T}}{\partial \varphi}\left(\varphi_{0}\right) \\
\tau_{e q}=\frac{\partial^{2} \tilde{\boldsymbol{w}}}{\partial \varphi \partial \epsilon_{e q}}\left(\varphi_{0}, \overline{\boldsymbol{\epsilon}}\right)=3 \epsilon_{e q} \frac{\partial G_{M T}}{\partial \varphi}\left(\varphi_{0}\right)
\end{array} .\right.
$$

The P-tensor (indirectly) in Eq. (23) is the classical P-tensor $\left(\mathbf{P}=\mathbf{S}:\left(\mathbf{C}^{\mathrm{MT}}\right)^{-1}\right)$ for spherical inclusions in an isotropic matrix where the reference stiffness is the Mori-Tanaka stiffness (C.1) (see e.g. Dormieux et al., 2006):

$$
\mathbf{P}=\frac{\alpha\left(\varphi_{0}\right)}{3 K_{M T}\left(\varphi_{0}\right)} \mathbf{J}+\frac{\beta\left(\varphi_{0}\right)}{2 G_{M T}\left(\varphi_{0}\right)} \mathbf{K}
$$




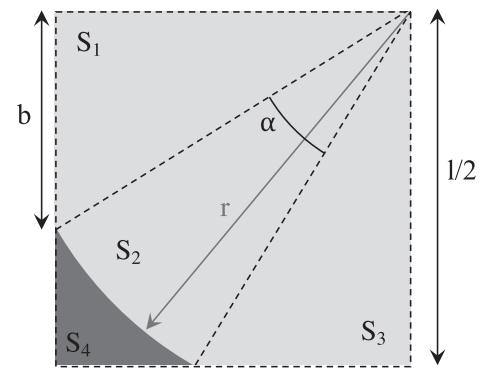

Fig. D.17. High porosity limit geometry.

with:

$$
\left\{\begin{array}{l}
\alpha\left(\varphi_{0}\right)=\frac{3 K_{M T}\left(\varphi_{0}\right)}{3 K_{M T}\left(\varphi_{0}\right)+4 G_{M T}\left(\varphi_{0}\right)} \\
\beta\left(\varphi_{0}\right)=\frac{6\left(K_{M T}\left(\varphi_{0}\right)+2 G_{M T}\left(\varphi_{0}\right)\right)}{5\left(3 K_{M T}\left(\varphi_{0}\right)+4 G_{M T}\left(\varphi_{0}\right)\right)}
\end{array} .\right.
$$

Eq. (23) can be simplified using $\mathbf{i}: \mathbf{J}: \mathbf{i}=3, \overline{\mathbf{e}}: \mathbf{K}: \overline{\mathbf{e}}=3\left(1 / 2+\epsilon_{m}^{2} / \epsilon_{e q}^{2}\right), \mathbf{i}: \mathbf{K}: \mathbf{i}=0, \overline{\mathbf{e}}: \mathbf{J}: \overline{\mathbf{e}}=0, \overline{\mathbf{e}}: \mathbf{J}: \mathbf{i}=0, \mathbf{i}: \mathbf{J}: \overline{\mathbf{e}}=0$, $\overline{\mathbf{e}}: \mathbf{K}: \mathbf{i}=0, \mathbf{i}: \mathbf{K}: \overline{\mathbf{e}}=0$, and reduces to:

$$
\tilde{\tilde{w}}\left(\sigma_{\varphi}, \bar{\epsilon}\right)=\frac{9}{2} \lambda_{e f f}\left(\varphi_{0}, \sigma_{\varphi}\right) \epsilon_{m}^{2}+\frac{3}{2} G_{e f f}\left(\varphi_{0}, \sigma_{\varphi}\right) \epsilon_{e q}^{2}+O\left(\sigma_{\varphi}^{3}\right),
$$

with $\lambda_{e f f}\left(\varphi_{0}, \sigma_{\varphi}\right)=\lambda_{M T}\left(\varphi_{0}\right)+\delta_{\lambda}\left(\varphi_{0}\right) \sigma_{\varphi}^{2} / 2$ and $G_{e f f}\left(\varphi_{0}, \sigma_{\varphi}\right)=G_{M T}\left(\varphi_{0}\right)+\delta_{G}\left(\varphi_{0}\right) \sigma_{\varphi}^{2} / 2$ the effective Lamé parameters. $\delta_{\lambda}\left(\varphi_{0}\right)$ and $\delta_{G}\left(\varphi_{0}\right)$ are given by:

$$
\left\{\begin{array}{l}
\delta_{\lambda}\left(\varphi_{0}\right)=\frac{\partial^{2} \lambda_{M T}}{\partial \varphi^{2}}\left(\varphi_{0}\right)+\frac{2 \alpha\left(\varphi_{0}\right)}{K_{M T}\left(\varphi_{0}\right)}\left(\frac{\partial \lambda_{M T}}{\partial \varphi}\left(\varphi_{0}\right)\right)^{2}+\frac{4 \beta\left(\varphi_{0}\right)}{3 G_{M T}\left(\varphi_{0}\right)}\left(\frac{\partial G_{M T}}{\partial \varphi}\left(\varphi_{0}\right)\right)^{2} \\
\delta_{G}\left(\varphi_{0}\right)=\frac{\partial^{2} G_{M T}}{\partial \varphi^{2}}\left(\varphi_{0}\right)+\frac{2 \beta\left(\varphi_{0}\right)}{G_{M T}\left(\varphi_{0}\right)}\left(\frac{\partial G_{M T}}{\partial \varphi}\left(\varphi_{0}\right)\right)^{2}
\end{array} .\right.
$$

The effective Young's modulus is thus deduced from,

$$
E_{e f f}\left(\varphi_{0}, \sigma_{\varphi}\right)=G_{e f f}\left(\varphi_{0}, \sigma_{\varphi}\right) \frac{3 \lambda_{e f f}\left(\varphi_{0}, \sigma_{\varphi}\right)+2 G_{e f f}\left(\varphi_{0}, \sigma_{\varphi}\right)}{\lambda_{e f f}\left(\varphi_{0}, \sigma_{\varphi}\right)+G_{e f f}\left(\varphi_{0}, \sigma_{\varphi}\right)}
$$

\section{Appendix D. High porosity limit of the ordered system with a spherical hole}

At high porosity, the region where stresses concentrate can be assimilated to an elastic beam of section $S(x)=4 S_{4}$ where $S_{4}=l^{2} / 4-S_{1}-S_{2}-S_{3}$ is the area of the shaded region in Fig. D.17. In this figure, $r$ is the radius of the circle at the intersection of the spherical pore and the plane $x$; that is, $r(x)=\sqrt{R^{2}-x^{2}}$. For $r \geq l / 2$, the surface areas $S_{1}, S_{2}$ and $S_{3}$ satisfy: $S_{1}=S_{3}=b l / 2$ with $b=\sqrt{r^{2}-l^{2} / 4}$ and $S_{2}=\alpha r^{2} / 2$ with $\alpha=\pi / 2-2 \arctan (2 b / l)$. Otherwise, when $r<l / 2$, it is $S_{1}=S_{3}=0$ and $S_{2}=\pi r^{2} / 4$.

\section{Appendix E. Local porosity measure for two-phase periodic solids}

Consider the unit-cell depicted on Fig. E.18 and the observation window $A_{1} A_{2} A_{3} A_{4}$ of side-length $l$. Each vertex $A_{i}$ belongs to a quarter-unit-cell containing a pore $\mathcal{P}_{i}$ of center $\left(x_{i}^{c}, y_{i}^{c}\right)$ and radius $R_{i}$. The local porosity measured in that unit cell is $\varphi_{a}=\left(S_{1}+S_{2}+S_{3}+S_{4}\right) / l^{2}$ where $S_{i}$ is the area of pore $\mathcal{P}_{i}$ intersected with the observation window. $S_{i}$ is a function of $\Delta x_{i}=x_{A_{i}}-x_{i}^{c}, \Delta y_{i}=y_{A_{i}}-y_{i}^{c}$ and $R_{i}$. Due to symmetries, $S_{2}(\Delta x, \Delta y, R)=S_{1}(-\Delta x, \Delta y, R), S_{3}(\Delta x, \Delta y, R)=S_{1}(-\Delta x,-\Delta y, R)$ and $S_{4}(\Delta x, \Delta y, R)=S_{1}(\Delta x,-\Delta y, R)$. Eleven cases (summarized in Fig. E.18) are to be considered for the evaluation of 


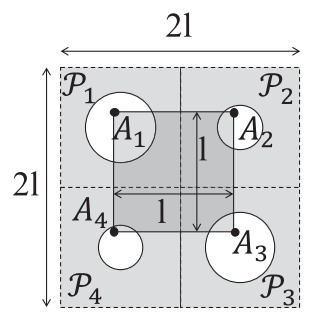

1.

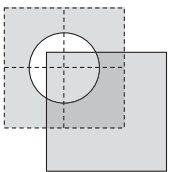

4.

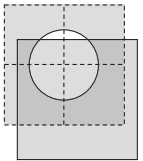

2.

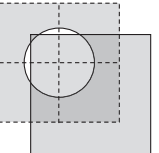

3.

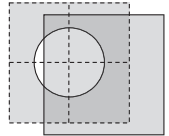

5.

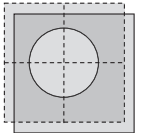

6.
7.
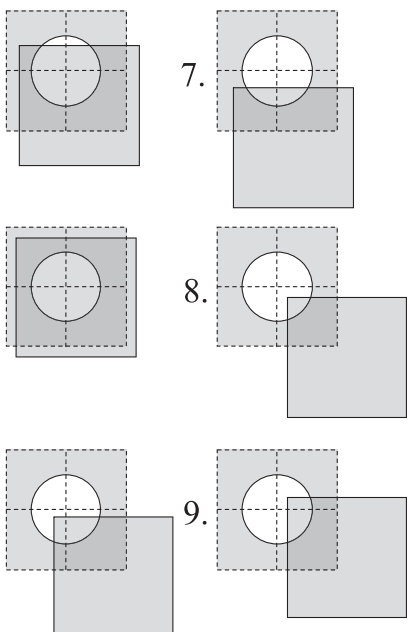

10

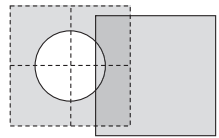

11.

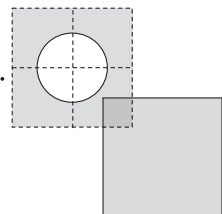

Fig. E.18. Calculation of the local porosity in a square observation window.

Table E.1

Eleven cases considered for the $S_{1}$ calculation.

\begin{tabular}{|c|c|c|c|c|c|c|c|c|c|c|c|c|c|}
\hline & $\Delta x \leq 0$ & $\Delta x \geq 0$ & $\Delta x \leq R$ & $\Delta x \leq-R$ & $\Delta x \geq-R$ & $\Delta y \leq 0$ & $\Delta y \geq 0$ & $\Delta y \leq R$ & $\Delta y \geq R$ & $\Delta y \geq-R$ & $\Delta x^{2}+\Delta y^{2} \leq R^{2}$ & $\Delta x^{2}+\Delta y^{2} \geq R^{2}$ & \\
\hline if & $x$ & & & & & & $x$ & & & & $x$ & & $S_{1}=S_{1}^{1}$ \\
\hline else if & $x$ & & & & & $x$ & & & & & $x$ & & $S_{1}=S_{1}^{2}$ \\
\hline else if & & $x$ & & & & $x$ & & & & & $x$ & & $S_{1}=S_{1}^{3}$ \\
\hline else if & & $x$ & & & & & $x$ & & & & $x$ & & $S_{1}=S_{1}^{4}$ \\
\hline else if & $x$ & & & $x$ & & & $x$ & $x$ & & & & & $S_{1}=S_{1}^{5}$ \\
\hline else if & $x$ & & & & $x$ & & $\times$ & & $x$ & & & & $S_{1}=S_{1}^{6}$ \\
\hline else if & $x$ & & & & $x$ & & $x$ & $x$ & & & & & $S_{1}=S_{1}^{7}$ \\
\hline else if & $x$ & & & $x$ & & & $x$ & & $x$ & & & & $S_{1}=S_{1}^{8}$ \\
\hline else if & $x$ & & & & & $x$ & & & & $x$ & & $x$ & $S_{1}=S_{1}^{9}$ \\
\hline $\begin{array}{l}\text { else if } \\
\text { else }\end{array}$ & & $x$ & $x$ & & & & $x$ & & & & & $x$ & $\begin{array}{l}S_{1}=S_{1}^{10} \\
S_{1}=S_{1}^{11}\end{array}$ \\
\hline
\end{tabular}

$S_{1}: S_{1}(\Delta x, \Delta y, R)=S_{1}^{i}(\Delta x, \Delta y, R)$ where $i$ is given in Table E.1 and the $S_{1}^{i}$ s are defined by:

$$
\left\{\begin{array}{l}
S_{1}^{1}(\Delta x, \Delta y, R)=\frac{3 \pi}{4} R^{2}+\frac{R^{2}}{2}\left(f\left(\frac{\Delta x}{R}\right)+f\left(\frac{\Delta y}{R}\right)\right)+|\Delta x \Delta y| \\
S_{1}^{2}(\Delta x, \Delta y, R)=\frac{\pi}{4} R^{2}+\frac{R^{2}}{2}\left(f\left(\frac{\Delta x}{R}\right)-f\left(\frac{\Delta y}{R}\right)\right)-|\Delta x \Delta y| \\
S_{1}^{3}(\Delta x, \Delta y, R)=-\frac{\pi}{4} R^{2}-\frac{R^{2}}{2}\left(f\left(\frac{\Delta x}{R}\right)+f\left(\frac{\Delta y}{R}\right)\right)+|\Delta x \Delta y| \\
S_{1}^{4}(\Delta x, \Delta y, R)=\frac{\pi}{4} R^{2}-\frac{R^{2}}{2}\left(f\left(\frac{\Delta x}{R}\right)-f\left(\frac{\Delta y}{R}\right)\right)-|\Delta x \Delta y| \\
S_{1}^{5}(\Delta x, \Delta y, R)=\pi R^{2}+R^{2} f\left(\frac{\Delta y}{R}\right) \\
S_{1}^{6}(\Delta x, \Delta y, R)=\pi R^{2}+R^{2} f\left(\frac{\Delta x}{R}\right) \\
S_{1}^{7}(\Delta x, \Delta y, R)=\pi R^{2}+R^{2}\left(f\left(\frac{\Delta x}{R}\right)+f\left(\frac{\Delta y}{R}\right)\right) \\
S_{1}^{8}(\Delta x, \Delta y, R)=\pi R^{2}- \\
S_{1}^{9}(\Delta x, \Delta y, R)=\quad-R^{2} f\left(\frac{\Delta y}{R}\right) \\
S_{1}^{10}(\Delta x, \Delta y, R)=\quad-R^{2} f\left(\frac{\Delta x}{R}\right) \\
S_{1}^{11}(\Delta x, \Delta y, R)=0
\end{array}\right.
$$

with $f(u)=|u| \sqrt{1-u^{2}}-\arccos |u|$.

\section{References}

Bayer, B., 1964. Relation between granularity and density for a random-dot model. J. Opt. Soc. Am. 54, 1485-1490.

Day, A., Snyder, K., Garboczi, E., Thorpe, M., 1992. The elastic moduli of a sheet containing circular holes. J. Mech. Phys. Solids 40, 1031-1051.

Del Gado, E., Ioannidou, K., Masoero, E., Baronnet, A., Pellenq, R., Ulm, F.-J., Yip, S., 2014. A soft matter in construction - statistical physics approach to formation and mechanics of C-S-H gels in cement. Eur. Phys. J. Spec. Top. 223, 2285-2295. 
Dormieux, L., Kondo, D., Ulm, F.-J., 2006. Microporomechanics. John Wiley \& Sons, Ltd.

Drach, B., Tsukrov, I., Trofimov, A., 2016. Comparison of full field and single pore approaches to homogenization of linearly elastic materials with pores of regular and irregular shapes. Int. J. Solids Struct. 96, 48-63.

Eshelby, J., 1957. The determination of the elastic field of an ellipsoidal inclusion, and related problems. Proc. R. Soc. A 241, $376-396$.

Fritsch, A., Dormieux, L., Hellmich, C., 2006. Porous polycrystals built up by uniformly and axisymmetrically oriented needles: homogenization of elastic properties. Comptes Rendus Mécanique 334, 151-157.

Fritsch, A., Dormieux, L., Hellmich, C., Sanahuja, J., 2009. Mechanical behavior of hydroxyapatite biomaterials: an experimentally validated micromechanical model for elasticity and strength. J. Biomed. Mat. Res. 881, 149-161.

Fritsch, A., Hellmich, C., Dormieux, L., 2010. The role of disc-type crystal shape for micromechanical predictions of elasticity and strength of hydroxyapatite biomaterials. Phil. Trans. R. Soc. A. 368, 1913-1935.

Fritsch, A., Hellmich, C., Young, P., 2017. Micromechanics-derived scaling relations for poroelasticity and strength of brittle porous polycrystals. J. App. Mech. $80,1-12$.

Gărǎjeu, M., Suquet, P., 2007. On the influence of local fluctuations in volume fraction of constituents on the effective properties of nonlinear composites. Application to porous materials. J. Mech. Phys. Solids 55, 842-878.

Hashin, Z., 1962. The elastic moduli of heterogeneous materials. J. App. Mech. 29, 143-150.

Hashin, Z., Shtrikman, S., 1962. A variational approach to the theory of elastic behavior of polycristals. J. Mech. Phys. Solids $10,343-352$.

Hellmich, C., Ulm, F.-J., 2002. Are mineralized tissues open crystal foams reinforced by crosslinked collagen? Some energy arguments. J. Biomech. 35, 1199-1212.

Herrmann, H., Roux, S., 1990. Statistical models for the fracture of disordered media. Random Materials and Processes. Elsevier Science.

Hill, R., 1965. A self-consistent mechanics of composite materials. J. Mech. Phys. Solids 13, 213-222.

Ioannidou, K., Krakowiak, K., Bauchy, M., Hoover, C., Masoero, E., Yip, S., Ulm, F.-J., Levitz, P., Pellenq, R., Del Gado, E., 2016. Mesoscale texture of cement hydrates. Proc. Natl. Acad. Sci. USA 113, 2029-2034.

Ishai, O., Cohen, L., 1967. Elastic properties of filled and porous epoxy composites. Int. J. Mech. Sci. 9, 539-546.

Lacey, P., 1954. Developments in the theory of particle mixing. J. Appl. Chem. 4, 257-268.

Laubie, H., Monfared, S., Radjaï, F., Pellenq, R., Ulm, F.-J., 2017a. Effective potentials and elastic properties in the lattice element method: isotropy and transverse isotropy. J. Nanomech. Micromech doi:10.1061/(ASCE)NM.2153-5477.0000125.

Laubie, H., Radjaï, F., Pellenq, R., Ulm, F.-J., 2017b. Stress transmission and failure in disordered porous media. Phys. Rev. Lett.. Under review

Lobb, C., Forrester, M., 1987. Measurement of nonuniversal critical behavior in a two-dimensional continuum percolating system. Phys. Rev. B 35, $1899-1901$.

Lu, B., Torquato, S., 1990. Local volume fraction fluctuations in heterogeneous media. J. Chem. Phys. 93, 3452-3459.

Markov, M., Kazatchenko, E., Mousatov, A., Pervago, E., 2012. Generalized differential effective medium method for simulating effective elastic properties of two dimensional percolating composites. J. App. Phys. 112, 1-4.

Masoero, E., Del Gado, E., Pellenq, R., Ulm, F.-J., Yip, S., 2012. Nanostructure and nanomechanics of cement: polydisperse colloidal packing. Phys. Rev. Lett. 109, 3-6.

Meille, S., Garboczi, E.J., 2001. Linear elastic properties of 2D and 3D models of porous materials made from elongated objects. Model. Simul. Mater. Sci. Eng. 9, 371-390.

Monfared, S., Laubie, H., Radjaï, F., Pellenq, R., Ulm, F.-J., 2017. Mesoscale poroelasticity of heterogeneous media. J. Nanomech. Micromech.. Submitted

Monfared, S., Ulm, F.-J., 2016. A molecular informed poroelastic model for organic-rich, naturally occurring porous geocomposites. J. Mech. Phys. Solids 88, $186-203$.

Mori, T., Tanaka, K., 1973. Average stress in matrix and average elastic energy of materials with misfitting inclusions. Acta Metall. $21,571-574$.

Norris, A., 1985. A differential scheme for the effective moduli of composites. Mech. Mater. 4, 1-16.

Sanahuja, J., Dormieux, L., Meille, S., Hellmich, C., Fritsch, A., 2010. Micromechanical explanation of elasticity and strength of gypsum: from elongated anisotropic crystals to isotropic porous polycrystals. J. Eng. Mech. 136, 239-253.

Smith, P., Torquato, S., 1988. Computer simulation results for the two-point probability function of composite media. J. Comput. Phys. 76, 176-191.

Suquet, P., 1997. Continuum Micromechanics. Springer-Verlag, New York.

Thorpe, M., Sen, P., 1985. Elastic moduli of two-dimensional composite continua with elliptical inclusions. J. Acoust. Soc. Am. 77, $1674-1680$.

Topin, V., Delenne, J.-Y., Radjaï, F., Brendel, L., Mabille, F., 2007. Strength and failure of cemented granular matter. Eur. Phys. J. E 23, 413-429.

Torquato, S., 2002. Random Heterogeneous Materials. Microstructure and Macroscopic Properties. Springer-Verlag, New York.

Torquato, S., Beasley, J., 1986. Effective properties of fiber-reinforced materials: II-Bounds on the effective elastic moduli of dispersions of fully penetrable cylinders. Int. J. Eng. Sci. 24, 435-447.

Wolfram ResearchInc, 2016. Mathematica. Version 10.1., Champaign, Illinois.

Zaoui, A., 2002. Continuum micromechanics: survey. J. Eng. Mech. 808-816. 